\title{
Particle and Particle-Surfactant Mixtures at Fluid Interfaces: Assembly, Morphology, and Rheological Description
}

\author{
Armando Maestro, ${ }^{1,2}$ Eva Santini, ${ }^{3}$ Dominika Zabiegaj, ${ }^{3}$ Sara Llamas, ${ }^{1,3}$ Francesca Ravera, ${ }^{3}$ \\ Libero Liggieri, ${ }^{3}$ Francisco Ortega, ${ }^{1}$ Ramón G. Rubio, ${ }^{1,4}$ and Eduardo Guzman ${ }^{1}$ \\ ${ }^{1}$ Departamento de Química Física I, Facultad de Ciencias Químicas, Ciudad Universitaria s/n, 28040 Madrid, Spain \\ ${ }^{2}$ Cavendish Laboratory, University of Cambridge, J.J. Thomson Avenue, Cambridge CB3 OHE, UK \\ ${ }^{3}$ Istituto per l'Energetica e le Interfasi, Consiglio Nazionale delle Ricerche, Via de Marini 6, 16149 Genova, Italy \\ ${ }^{4}$ Instituto Pluridisciplinar, Universidad Complutense de Madrid, Avenida Juan XXIII 1, 28040 Madrid, Spain
}

Correspondence should be addressed to Armando Maestro; am2212@cam.ac.uk, Eva Santini; e.santini@ge.ieni.cnr.it, and Eduardo Guzman; eduardogs@quim.ucm.es

Received 19 April 2015; Revised 5 August 2015; Accepted 6 August 2015

Academic Editor: Charles Rosenblatt

Copyright ( 2015 Armando Maestro et al. This is an open access article distributed under the Creative Commons Attribution License, which permits unrestricted use, distribution, and reproduction in any medium, provided the original work is properly cited.

\begin{abstract}
We report here a review of particle-laden interfaces. We discuss the importance of the particle's wettability, accounted for by the definition of a contact angle, on the attachment of particles to the fluid interface and how the contact angle is strongly affected by several physicochemical parameters. The different mechanisms of interfacial assembly are also addressed, being the adsorption and spreading the most widely used processes leading to the well-known adsorbed and spread layers, respectively. The different steps involved in the adsorption of the particles and the particle-surfactant mixtures from bulk to the interface are also discussed. We also include here the different equations of state provided so far to explain the interfacial behavior of the nanoparticles. Finally, we discuss the mechanical properties of the interfacial particle layers via dilatational and shear rheology. We emphasize along that section the importance of the shear rheology to know the intrinsic morphology of such particulate system and to understand how the flow-field-dependent evolution of the interfacial morphology might eventually affect some properties of materials such as foams and emulsions. We dedicated the last section to explaining the importance of the particulate interfacial systems in the stabilization of foams and emulsions.
\end{abstract}

\section{Introduction}

The assembly of micro- and nanoparticles at fluid interfaces, both liquid/vapor and liquid/liquid, has become a topic with increasing interest in recent years due to the many theoretical and practical implications associated with these systems [1-3]. This is associated with the ubiquitous presence of particles trapped at fluid interfaces in nature and in technological processes [4-8]. Beyond their interest in technological applications such as the stabilization of dispersed systems (foams, emulsions, or thin films), flotation processes, encapsulation, pharmaceutical formulations, or food technology [9-17], particles at fluid interfaces present a more fundamental interest because they provide good models for studying physicochemical features of interfacial soft systems such as their interaction potentials, structure, and rheology $[3,18,19]$. Furthermore, the increasing development of the processes for fabrication of nanoparticles based materials has raised important questions related to the potential risks and hazards associated with the interactions of nanoparticles with the environment [20-22] and with cells and tissues [23-30]. These toxicological aspects of particles are generally associated with their interactions with fluid interfaces. Thus, the understanding of the interaction of particles at fluid interfaces has been focused on the efforts of many researches in the last thirty years, becoming an important challenge for both academia and industry in the development of new products and processes [1-4, 31, 32]. Despite the extensive research on this type of systems many questions such as the processes involved in the particle 
adsorption or the relationship between the structure and properties of the interfacial layers formed by particles remain poorly understood yet $[1,3]$.

The main difficulty for the comprehensive understanding of the formation processes and properties of particle-laden interfaces is associated with the complex interplay between different physicochemical-chemical aspects such as particle wettability, size, shape, surface charge, and chemical nature $[4,32]$. These aspects govern the attachment of particles to the fluid interfaces in such a way that in many cases it is difficult to be quantified, thus leading to complex scenario in which many forces operate synergistically.

One of the difficulties associated with the understanding of particle-laden interfacial layers is their multiphase character where three different interfaces can be identified, being one of them a fluid-fluid interface, that is, liquid/vapor or liquid/liquid, whereas the other two are two solid-fluid interfaces. The definition of the interface as a multiphase region can be easily understood by the schematic representation in Figure 1(a) [1, 33, 34].

The attachment of particles to fluid interfaces leads to the formation of particle assemblies that modify the mechanical stability of the interfaces, providing the bases for hindering the drop/bubble coalescence. This important property of particle-laden interfaces has been developed from the pioneering works by Ramsden [35] and Pickering [36] for the stabilization of bubbles, thin films, or dispersed systems [5, 13]. However, there still is a poor knowledge on the governing laws of the stabilization phenomena. Thus, several questions have been raised in the last years in relation to the role of particles in the stabilization of interfaces. Among them, the understanding of the energies involved in the particles attachment to fluid interfaces is probably one of the most important ones since they play a central role in determining the reversibility and irreversibility of the particle attachment to fluid interfaces. Another aspect to understand is the role of particle layers in the stabilization of interfaces, that is, the mechanical performance of particle-laden interfaces [37-39]. This is critical because most of particulate interfacial systems undergo shear and dilational deformations under flow [32]. Thus, particle layers must provide enough mechanical resistance to guarantee the stabilization of drops and bubbles under mechanical tension conditions. However, a strong controversy has been recently raised in this topic and many questions remain still open [40-43]. This is related to the role in the stabilization of the dispersed systems of aspects such as the particle distribution at the interface, particle contact angle and morphology, or even the aggregation degree of these particles that makes rationalizing the real role of the mechanical stability of the particle-laden interface difficult [1].

It is also important to consider the role of the morphology of the interface in their mechanical stabilization by the attachment of particles. This plays a central role because many of the technological applications of systems involving the interaction of particles with fluid interfaces require designing structures with a defined geometry $[1,18$, 19, 32]. Furthermore, it has been found that particles at fluid interfaces can be distributed forming a wide variety

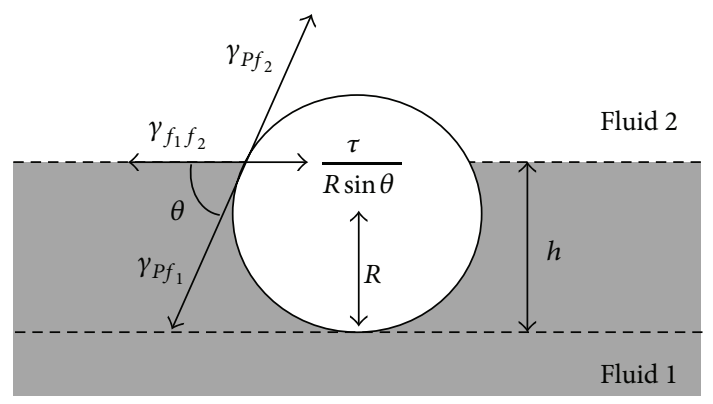

(a)

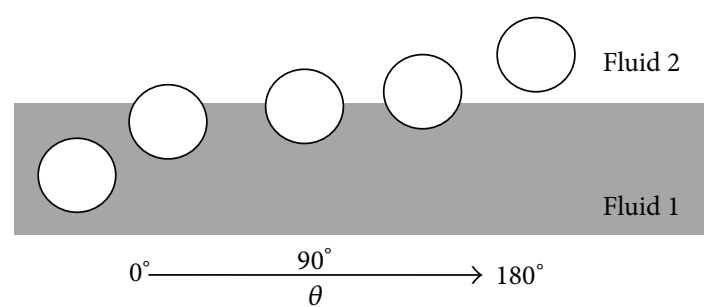

(b)

Figure 1: (a) Sketch of the contact angle, $\theta$, for a particle at a fluid interface. $\gamma_{P f_{1}}$ is the interfacial tension particle-bottom fluid, $\gamma_{P f_{2}}$ the interfacial tension particle-upper phase, $\gamma_{f_{1} f_{2}}$ the interfacial tension fluid-fluid, and $\tau$ the line tension associated with the contact line of the particle with the interface. (b) Sketch of the position of a particle, with increasing hydrophobicity at the interface. Reproduced from [2], Copyright 2014, with permission from Elsevier.

of structures ranging from perfect $2 \mathrm{D}$ crystal to amorphous patterns of aggregated particles [18, 19]. Thus, it is expected that the presence of different interfacial structure can affect the mechanical performance of the layers and their collapse mechanism with the appearance of buckling, jamming, or expulsion phenomena in the particle-layer interfaces $[32,44$, $45]$. In this context, the understanding of the interactions involved in particle-laden interfaces plays a central role due to their importance in the control of the structural features of particle layers and in the film properties (optical, mechanical, etc.) $[46,47]$.

This paper presents an overview of the most recent advances in the understanding of particle-laden layers. We focus our attention on the forces involved in the particle attachment to the fluid interface and on the effect of particles on the mechanical performance of interface. We also address how the stabilization of dispersed systems can be enhanced using particulate systems, emphasizing the correlation of the destabilization processes with the aforementioned parameters.

\section{Attachment of Particles to Fluid Interfaces: Contact Angle and Trapping Energy}

Two different routes allow the particles to arrive to the interface [51]: the direct interfacial assembly from bulk dispersions forming an adsorbed monolayer [52, 53] or the direct spreading of controlled amount of particles at the fluid interface from particles dispersions in a volatile organic 
solvent to form spread monolayers [18, 19]. It is worth mentioning that in the former case the density of particles at the interface is governed by the tendency to segregate of the particles to the interface, which depends on their bulk concentration, whereas in the latter case the density can be easily controlled from the initial spread amount. On the other side, in order to address fundamental physicochemical aspects of particle-laden interfaces (strongly dependent on the interfacial morphology), spread monolayers are preferred because spreading is always faster and allows a fine control of the surface $[18,19]$.

Independently of the type of monolayers considered, the attachment of particles to fluid interfaces depends on physicochemical parameters such as size, chemical nature, particle surface roughness, and wettability. The latter is probably the most important one, being evaluated by the contact angle $\theta . \theta$ is associated with the balance of energies involved on the particles attachment to the fluid interface according to the scheme shown in Figure 1(a).

A qualitative description of $\theta$ can be obtained by defining the partition of the particles between the different fluid phases (Figure 1(a)); thus it can be considered that $\theta$ plays a role similar to the hydrophilic-lipophilic balance (HLB) of surfactants $[6,54]$. An arbitrary description of the hydrophilicityhydrophobicity of the particles defines $\theta=90^{\circ}$ as the threshold value of the contact that separates their hydrophilic $\left(\theta<90^{\circ}\right)$ and their hydrophobic $\left(\theta>90^{\circ}\right)$ characteristics. There are two limits, $\theta=0-10^{\circ}$ and $\theta=170-180^{\circ}$, for which the particles are preferentially distributed in one of the two fluid phases. Figure 1(b) shows schematically the effect of $\theta$ on the relative position of the particles at the interfacial plane. This relative position of particles at the interfacial plane affects the interactions along the interface that can modify the particle functionality as stabilizing agent of the interfaces. The above discussion leads to considering that the attachment of particles at fluid interfaces can be easily described by $\theta$ (and the particle radius) or, eventually, by the particle flotation height $h$ that defines the perimeter of the contact line (see Figure 1(a)). In view of the relation between $\theta$ and $h$, the former is known to be strongly dependent on the line tension $\tau$ [47]. $\tau$ is defined as the excess free energy associated with the line where the particle and the two fluids meet, according to Gibbs [47]. It is negligible for colloidal microparticles, whilst it has a nonnegligible role in controlling the attachment energies of nanoparticles to the fluid interfaces. Thus, the contributions of the line tension to the particle attachment at fluid interface become important in the nanoscale due to the role of several aspects such as the heterogeneity in surface roughness and chemical nature of the particles in the modification of the wettability of the particles in relation to that of macroscopic surfaces $[55,56]$. The role of the line tension decreases rapidly with the increase of the particle size as was pointed out by Isa et al. [55].

The definition of $\theta$ for particles at fluid interfaces, including the effect of the line tension, can be easily obtained by the modified Young equation [57-59]:

$$
\frac{\left(\gamma_{P f_{2}}-\gamma_{P f_{1}}\right)}{\cos \theta}-\gamma_{f_{1} f_{2}}=-\frac{\tau}{R \sin \theta},
$$

where $\gamma_{P f_{2}}$ is the interfacial tension particle-upper phase, $\gamma_{P f_{1}}$ is the interfacial tension particle-bottom phase, $\gamma_{f_{1} f_{2}}$ is the interfacial tension between the fluid phases, $R$ is the radius of the particle, and $\tau$ is the line tension. The line tension decreases the contact angle in relation to that expected for a macroscopic wetted surface [60]. Furthermore, it is important to consider the important role played for the line tension in the energetic landscape of particle-laden interface, controlling the transition wetting-drying of the particles for the fluid interface [60], which was also evidenced independently by Bresme and Quirke using molecular simulation [61]. However, they found values for the line tension that were at least one order of magnitude higher than those obtained previously from experimental data [60]. This discrepancy might be associated with the different line tension dependence on the surface tension between the fluid phases and the solid, obtained via simulations and experiments.

Despite the important role of the line tension on the particles attachment, for the sake of simplicity no further discussion on the role of the line tension will be included [62]. Thus, the contact angle of particles attached to fluid interfaces can be simply expressed by neglecting $\tau$ in (1) yielding

$$
0=\gamma_{P f_{2}}-\gamma_{P f_{1}}-\gamma_{f_{1} f_{2}} \cos \theta
$$

When $\gamma_{P f_{2}}>\gamma_{P f_{1}}$ particles are hydrophilic $(0 \leq \theta<90)$, whereas they are hydrophobic $\left(90<\theta^{\circ} \leq 180\right)$ for $\gamma_{P f_{2}}<\gamma_{P f_{1}}$. It is obvious at this point that the principal handicap to get reliable values of $\theta$ comes from the measurement of the fluidsolid interfacial tensions.

The complete understanding of the attachment of particles to fluid interfaces requires the evaluation of the energies involved in the process. Thus, the total energy associated with the attachment of particles to the fluid interface can be obtained by the difference between the energies of the particle at the interface and in the bulk suspension. For the simplest case that considers small particles attached to an arbitrary interface and small enough for the gravity effects being negligible, the attachment energy, $\Delta E_{p}$, is given by

$$
\Delta E_{p}=-\pi R^{2} \gamma_{f_{1} f_{2}}(1 \pm \cos \theta)^{2} .
$$

The \pm sign allows identifying the relative position of the centre of the particle in relation to the interfacial plane. Indeed, (+) represents the situation of those particles with their centre above the interfacial plane, whereas (-) represents the situation of particles with the centre below the interfacial plane. The formation of interfacial layers of conventional surfactants, the adsorption-desorption equilibrium takes place in time scales ranging between milliseconds and several seconds. However, it is known that the attachment of particles to fluid interfaces can be considered in most cases an irreversible process, being the energies involved in the attachment many times the thermal energy $\left(k_{B} T\right.$, being $k_{B}$ the Boltzmann constant and $T$ the temperature). As a consequence, once the particles arrive to the interface they remain trapped in a quasi-2D layer, and only small fluctuations from the equilibrium positions in the direction perpendicular to the interfacial plane are expected due to 
thermal or capillary deformations of the interface. This does not mean that once the particles arrive to the interface they remain attached at fixed positions; the real scenario implies the continuous diffusion of the particles along the $2 \mathrm{D}$ plane in addition to the small fluctuations above and below the interfacial plane. Equation (3) points out that the reversibility of the particles' attachment to the fluid interfaces is mainly governed by $R$ and $\theta$. For instance, for spherical particles with a fixed contact angle around 60 degrees adsorbed at an arbitrary interface with a interfacial tension of $50 \mathrm{mN} / \mathrm{m}$, the attachment energy is around $10^{7} k_{B} T$ for the case of particles with a radius around $1 \mu \mathrm{m}$. This energy decreases to values in the order of $10^{4} k_{B} T$ when the size is reduced till $10 \mathrm{~nm}$. In this context, it is possible to assume that the microparticles are usually irreversibly attached to the fluid interfaces whereas for nanoparticles the reversibility of the adsorption can be tuned by the modification of the contact angle $\theta$ [2]. The reversibility-irreversibility of particles attachment at fluid interfaces was addressed both theoretically and experimentally by Wi et al. [63], who pointed out that the attachment to the interface of particles larger than $10 \mathrm{~nm}$ can be considered completely irreversible. However, for smaller particles, a true thermodynamic equilibrium between the bulk phases and the interface is established, with the line tension playing a central role in the energetic landscape. It is worth mentioning that the role of the reversibility or irreversibility of particles attachment to fluid interfaces is essential for controlling the structure of interfacial assemblies of particles because they have a central role in the particle interactions at the interface and in the layer response to external mechanical perturbations, for example, collision between droplets in an emulsion [64].

In the last twenty years, several experimental techniques have been developed for the determination of the contact angle of particles at fluid interfaces: drop shape techniques $[56,65,66]$, surface pressure-area isotherms $[52,67-70]$, Washburn capillary rise method [71-73], Atomic Force Microscopy (AFM) coupled to colloidal probe [74, 75], Gel Trapping Technique (GTT) [56, 67, 76, 77], Freeze Fracture Shadow Casting (FreSCa) [55, 78-80], excluded area method [81, 82], Film-Calliper Method (FCM) [83], ellipsometry [53, 84-88], or neutron reflectivity [89]. It has been found that different methods to evaluate the contact angle of particles provide different values of $\theta$ for similar particles [2]. This is due to the different assumptions used to calculate the contact angle from the raw data. A detailed discussion of the different methods for the determination of the contact angle of particles attached to fluid interfaces was presented by Maestro et al. [2] in a recent review. One of the most important problems related to the determination of the contact angle of particles at fluid interfaces comes from the type of contact angle evaluated (advancing or receding). This aspect is not clear for most of the methods used for the determination of $\theta$.

Many variables can modify the wettability and consequently the attachment energy of particles at fluid interfaces. The most important of them is probably the HLB of the particles, which is affected by the chemical nature of both particles and of the two phases adjacent to the interface. This balance can be easily tuned either by physical or chemical medications of the particles surfaces. In the former case, species able to modify the surface activity of the particles (e.g., common surfactant or short-chain alcohols) are attached to the surface of the particle by electrostatic, hydrogen bond, or van der Waals interactions, whereas in the latter case the modification of the nature of the particle's surface is performed by a chemical functionalization (e.g., thiolization of gold particles or silanization of silica surfaces) [86]. An alternative method, with increasing interest nowadays, is the fabrication of particles with asymmetrical wettability, the socalled Janus particles $[4,90,91]$.

The effect of the particles size on the contact angle of particles is accounted for among the most studied effects. In most of the cases, $\theta$ increases with the particle sizes till a constant value. This is generally explained considering a change in the chemical nature of the particle's surface as the size increases that confers a different hydrophobicity degree to the particles of different sizes [83]. Finally, $\theta$ reaches a plateau at a certain size due to a transition between nanoscopic wetting to macroscopic one, which is determined by the contribution of $\tau$ to the attachment of the particles to the interface $[55,56]$. McBride and Law pointed out that, for silica particles larger than $60 \mathrm{~nm}$, their contact angle at a liquid polystyrene/vapour interface assumed values similar to those found for the contact angle of a liquid polystyrene drop onto a macroscopic surface with the same chemical nature than the particles [56]. This can be interpreted in terms of a constant contribution of the line tension to the particle wetting once a threshold value for the particle size is reached in agreement with the results by Isa et al. [55].

The surface charge density of the particles is also a critical parameter to control $\theta$ because it affects the complex balance of interactions occurring at the interface and governs the stabilization of particles in their position at the interface [92, 93]. Several authors have pointed out that a decrease of the effective charge density of the particles leads to the increase of the contact angle of the particles [77, 93]. The reason is the partial hindering of the electrostatic barrier associated with the repulsion of the particles, which plays a central role in the control of the particle adsorption. Additionally, it is worth mentioning that $\theta$ is mainly governed by the interactions occurring along the nonpolar phase, as evidenced by the negligible effect on the contact angle motivated by the increase of the ionic strength. In this context, the differences of dielectric constant between the two fluid phases, and consequently the chemical nature of the fluid interface, determine the attachment of particles at the interface due to the existence of image-particle interactions associated with the presence of particles close to the two-phase boundaries. Thus, electrodipping forces drive the particles towards the phase with higher dielectric constant. This fact provides the bases for explaining the higher contact angle found for the same particles at the water/oil than at the water/air $[53,77]$.

The chemical nature of the particles surface plays also a central role in the wettability control. The chemical modification of silica particles by silanization was found to 
increase the value of $\theta$ by a factor close to 2 when the number of percentages of free silanol groups was reduced from $34 \%$ to $20 \%$ [86]. Similar effects were found by chemical grafting of poly(glycerol-monomethacrylate) chains to polystyrene latex particles. The increase of the length of the grafted chains leads to the decrease of the contact angle of particles at both air/water and n-dodecane/water interfaces due to the enhanced hydrophilicity of the particles [94]. The wettability of the particles can be also modified by the physical adsorption of surfactant to the surface of the particles as was evidenced by Maestro et al. [53], who studied the effect of two alkyltrimethylammonium bromides (hexadecyl-trimethylammonium bromide, CTAB and dodecyl-trimethylammonium bromide, DTAB) on the contact angle at the air/water interface of completely hydrophilic silica nanoparticles. They pointed out that a complex balance between the hydrophobic and the electrostatic interactions established in the mixed particle-surfactant system governs the contact angle. Thus, the concentration of surfactant in the solution plays a key role in the control of the particle wettability. The results also evidenced that the maximum value for the contact angle of the particles is associated with the neutralization of the particle surface charge by the adsorption of surfactant molecules. Further increases of the surfactant concentration lead to the rehydrophilization of the particles and consequently to the contact angle decrease. A similar behavior was found by Binks et al. [78] for the adsorption of mixtures of silica particles and a surfactant with two tails (didecyldimethylammonium bromide). Also, the wettability can be modified by small molecules such as alcohols [67, 91]. This latter effect was attributed to the formation of solvent layers onto the particle surface which modify their surface nature and consequently the contact angle at the fluid interfaces. Following with the role of the particle surface nature on the contact angle of particles at fluid interfaces, it is important to consider the role of roughness and porosity; these two features play a more important role as the particle size decrease [21,67]. The increase of the roughness and porosity of the particles leads to the increase of the contact angle in such a way that can be qualitatively explained assuming a classical Cassie-Baxter model [95]. This is related to the existence of asymmetric wetting along the contact line, thus defining different pinning-depinning phenomena of the particles at the interface. Furthermore, the roughness and porosity of the particles can influence the adsorption of surface active modifiers onto the particles. Theoretical calculations by Nonomura and Komura [95] showed that the wetting of particles depends on the interfacial tensions between the particles and the liquid phases, the particle sizes, and the fraction of the surface area of the particle that is in contact with the external liquid phase. This later leads to the asymmetric wetting of the particles due to their roughness, which can be well described on the bases of the Cassie-Baxter model, especially for those particles with a high roughness. Despite the potential importance of these aspects in the contact angle of particles at fluid interface, no systemic studies on their role have been addressed.
Additionally, the effect of the methodology use for the preparation of the particle-laden interface cannot be neglected in the understanding of the contact angle of particles at fluid interfaces [39]. For those particle layers obtained from the adsorption of the particles from bulk dispersions to the interface, a subtle balance of energies is expected (interactions fluid-fluid and fluid-particles), which determines the most probable contact angle of the particles at the interface. On the contrary, for spread monolayers, the particles are directly deposited at the interface independently of their contact angle.

\section{Surface Tension and Structure of Particle-Laden Interfaces}

The HLB is one of the most important parameters governing the position of particles at the interface. However, in contrast to that occurring for surfactant in which the HLB determines the portioning of the molecules between the interface and the two adjacent fluids phase, in most cases for particleladen interfaces once the particles arrives to the interface they remain trapped irreversibly and no partitioning of the particles between the fluid phases can be expected due to the high attachment energies involved, which overcome many times the thermal energy as was discussed above [96]. Thus, the classical equations of state (Langmuir, Frumkin, etc.) that described the thermodynamic behavior of interfacial layers formed by surfactants do not provide an accurate description of the relationship existing between the interfacial tension, the bulk concentration, and the surface coverage of particle layers [97]. In order to overcome these problems for the thermodynamic description of the particle-laden interface, new models considering their specific features have been developed in recent years $[48,98]$.

The first attempt to provide a thermodynamic description of particle-laden interface was performed by Binks [6] on the bases of the Volmer and van der Waals equations. The model considered that each particle behaves as common surfactant molecule. However, the model fails and provides unrealistic dependences of the surface tension on the interfacial coverage. This is explained taking into account the big difference that exists between the behavior of particles and common surfactant at the interfaces, and the different interactions at the interface. Additionally, the different length scales for particles and common surfactants are expected to play a key role in the thermodynamic behavior of particleladen interface, and a correct thermodynamic model must consider this fact. Miller et al. [98] developed a model for the description of particle-laden interfaces following theoretical approaches similar to the ones previously used for protein layers [99]. Using several assumptions the model provides an expression for the surface pressure $\left(\Pi=\gamma_{0}-\gamma\right.$, with $\gamma$ being the surface tension of the particle-laden interface and $\gamma_{0}$ the surface tension of the pure fluid interface)-area isotherm $[48,98]$ that reads as follows:

$$
\Pi=-\frac{k_{B} T}{\omega_{0}}\left[\ln \left(1-\frac{\omega}{A}\right)+\left(\frac{\omega}{A}\right)\right]-\Pi_{\mathrm{coh}},
$$




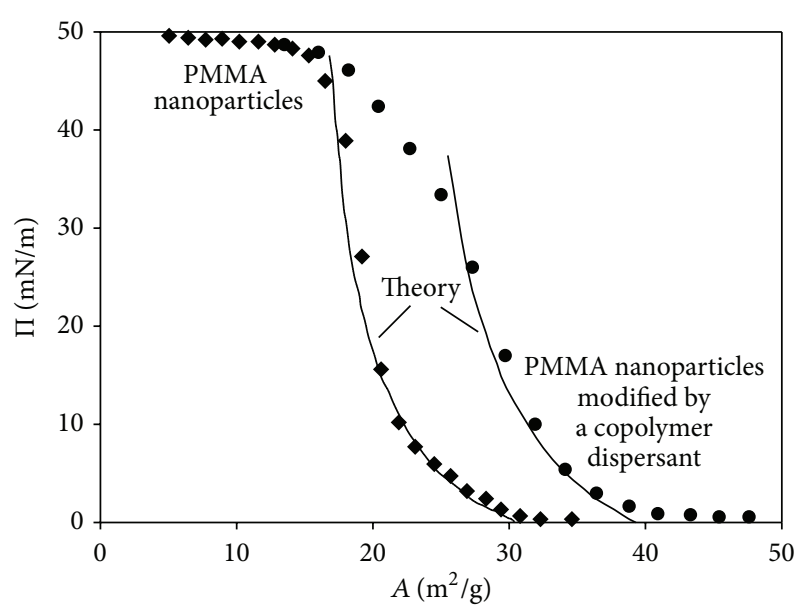

FIgURE 2: Comparison of experimental and theoretical isotherms for bare polymethylmethacrylate (PMMA) particles and PMMA particles with the surface modified with a copolymer. See the qualitative agreement between the experimental results and the theoretical model described by (4). The size of the particles was $113 \mathrm{~nm}$. Reprinted with permission from [48]. Copyright 2006, American Chemical Society.

where $\omega / A$ accounts for the interfacial coverage and $\omega_{0}$ is the area of a particle. Consider that

$$
\Pi_{\mathrm{coh}}=\frac{k T}{\omega_{0}} a\left(\frac{\omega}{A}\right)^{2}
$$

is the so-called cohesion pressure that is related to the balance of energies existing at the interface, depending strongly on the contact angle of the particles at the fluid interface, and accounts for the organization and degree of packing of the particles at the interface. This model predicts a thermodynamic behavior rather independent of the particle size and the nature, providing a good description for the behavior of both soft and hard particles. Figure 2 shows some examples of the application of the aforementioned thermodynamic model to experimental isotherms. These results pointed out a rather good agreement between the experimental results and the theoretical model before the collapse of the monolayer at lower areas. However, the validity of this model has been tested with a small number of systems so far.

Basically, most of the research focused on the steady state behavior of particle-laden interface is based on surface tension-surface concentration isotherms. However, this is not easily evaluable for particle-laden interfaces, and only when the particles are directly spread at the interface it is possible to obtain this type of relations. Several authors have given results of the steady state of particle-laden interfaces [33, 43, $53,100,101]$; however a description of the experimental results in terms of theoretical models, such as Frumkin or Langmuir, is difficult and no systematic studies are available. Figure 3 shows the experimental surface tension isotherms obtained for palmitic acid (PA) at the water/hexane interface and the mixture of PA and silica nanoparticles at the same interface $[49,50]$. The results pointed out that for the pure palmitic acid

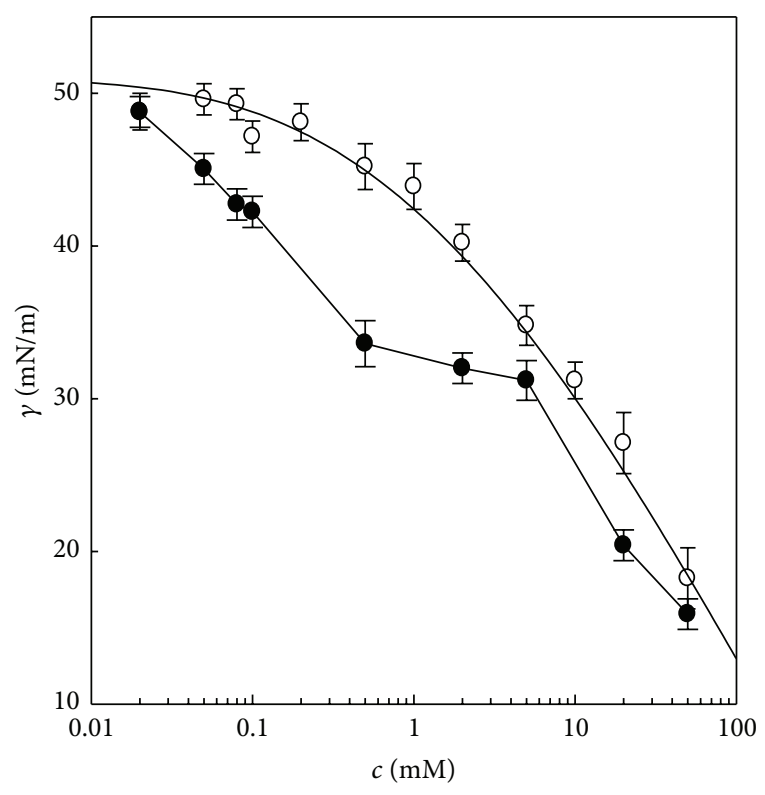

FIGURE 3: Dependences of the surface tension of the water/hexane interface on the PA concentration: PA $(O$, the solid lines represent the fitting of the experimental data to the Frumkin adsorption isotherm) and silica nanoparticles-PA mixtures $(\bullet)$. The particle concentration in the bulk was $1 \mathrm{wt} \%$. Reproduced from [49], Copyright 2014, with permission from Elsevier.

Frumkin's model describes well the experimental isotherm. On the contrary, a theoretical description of the isotherm of the system containing particles is rather difficult. Furthermore, the results for the PA-silica system at the water/hexane interface showed a strong synergetic effect on the reduction of the surface tension in relation to that observed for the pure PA solutions. Similar synergetic effect has been observed for other types of mixtures such as protein-surfactant and polyelectrolyte-surfactant systems $[22,102,103]$. It is worth mentioning that the bare silica particles do not present any surface activity and a surface tension value close to that of pure water was found for them independent of their bulk concentration as was discussed previously by Ravera et al. [33].

The comparison of mixed dispersion of PA and silica nanoparticles with this dispersion in which silica nanoparticles are combined with conventional surfactant such as $\mathrm{CTAB}$ and DTAB showed strong differences in the ability to produce surfactant decorated nanoparticles depending on the surfactant nature, whereas for CTAB or DTAB decorated silica nanoparticles an almost negligible change on the surface tension for the lowest surfactant concentrations was observed due to the low interfacial coverage; a higher efficiency of PA was found for the preparation of surfactant decorated nanoparticles with interfacial activity $[33,34,37]$. Thus, it is possible to assume that ionic surfactants are less efficient for modifying the hydrophobicity of particles than fatty acids (such as PA). Increasing the surfactant concentration, the effectiveness of the ionic surfactant to increase the hydrophobic character of the complexes is enhanced, favoring their 
attachment at the interface. An additional contribution to the incorporation of the particles to the interface can be associated with the interaction of the hydrophobic tails of the surfactant decorated particles at the interface $[53,100,104]$. Maestro et al. [53] pointed out that the length of the surfactant chains does not play an important role in the control of the wettability properties of the particles.

The study of the interaction of carbon soot particles with anionic surfactant has pointed out the absence of appreciable modifications of the pure surfactant layer due to the presence of carbon particulates. This is ascribed to the fact that carbon is strongly hydrophobic and the surfactant interaction occurs by hydrophobic interactions, thus leading to the hydrophilization of the particles. The consequence is a depletion of surfactant that is expected from the interface due to its interaction with the particles, but no particle incorporation to the interface occurs [100]. The results found for carbon soot-anionic surfactant mixtures contrast with those found for silica nanoparticles - trimethylalkylammonium bromides ones. In this latter case, there is a depletion of surfactant molecules from the bulk dispersion due to their interaction with the particles and the subsequent adsorption onto their surface. Therefore it is expected that only a residual amount of surfactant remains free in the solution $[33,34]$, explaining the decrease of the interfacial tension due to the attachment of particle-surfactant complexes at the fluid interface rather than the adsorption of free surfactant molecules, as it is expected for the aforementioned systems containing carbon soot [100]. The complex scenario found for the formation of particle-laden layers obtained by mixtures of particles and surfactant contrasts with that found for the formation of layers with polymer grafted particles, where the chemical nature of the polymer chains controls the particle adsorption [105].

The situation for the oil/water interface is different. In this case, the typical phenomena found for the air/water interfaces are enriched because of the appearance of additional process such as the transference of bare particles and surfactant molecules and/or particles-surfactant complexes between the two fluid phases $[38,50]$. This can affect the synergetic effect of the particle-surfactant interaction as was shown by Santini et al. [49].

In order to obtain a complete description of particle-laden interfaces it is necessary to characterize the morphological and structural features of the interfacial films. Information on these aspects can be obtained by two different approaches: the first one is based on the analysis of distribution of particles along the quasi-2D layer, that is, the determination of the interfacial morphology of the particle-laden interface. The second one is based on the analysis of the position of the particles with respect to the adjacent phases, which is related to the contact angle of the particles.

Several authors have shown that the organization of complexes at the interfacial layers depends on the packing density $[50,52]$ and therefore on the coverage of the interfacial layer. For silica nanoparticles-CTAB layers Santini et al. found by using Brewster Angle Microscopy (BAM) that, depending on the hydrophobicity of the complexes, the interfacial coverage is modified and this affects the interfacial textures of the layers [52]. This can be rationalized considering the effect of the surfactant concentration on the incorporation of the particles to the interface [53], so that the number and size of the interfacial aggregates increase till the formation of a close packed film. Similar behavior was found for PA-silica nanoparticles systems adsorbed at the air/water interface [50]. This system showed the formation of isolated particle islands, at the lowest surfactant concentration, that coalesce with the increase of the PA concentration to form a closepacked particle monolayer (see Figure 4).

Additional information on the organization of particles at the interface can be obtained from ellipsometry experiments. Maestro et al. [53] pointed out that the relative position in relation to the interfacial plane of silica nanoparticles can be easily tuned by the addition of alkyltrimethylammonium bromide in different concentrations. Similar results were found for the effect of the degree of silanization on silica particles by Zang et al. [86]. Thus, it is possible to assume that the wettability of the particles determines their position in relation to the interfacial plane. Santini et al. [50] studied by ellipsometry the thickness of PA-silica nanoparticles layers as function of the PA concentration. Their results pointed out clearly that the increase of the surfactant concentration leads to a transition from a low packed film (thickness several times smaller than the particle diameter) to a closed packed monolayer with a thickness comparable to the particle diameter. This shows a good agreement with the BAM images.

\section{Dynamics of the Particle Incorporation to Fluid Interfaces}

The adsorption of particles to fluid interfaces is a dynamic process governed by an intricate balance of interactions that determines the final position of the particles at the interface. The transference of the particles from the bulk dispersion to the interface can be governed by simple diffusion, but in some cases additional contributions due to the presence of interfacial energy barriers to the adsorption must be considered for the comprehensive description of the adsorption process [106]. When the adsorption of particles to fluid interfaces is investigated by the changes of the interfacial tension, the time scales involved in the adsorption process can be up to 3 orders of magnitude higher than for surfactant adsorption $[33,38]$.

The transference from bulk dispersion to the interface is generally a bimodal process $[33,37,53]$ with a fast step governed by the diffusion of the particles to the interface, being the characteristic time of this process comparable to that for the diffusion in bulk for particles of the same size. This time is not significantly affected by the hydrophobicity of the particles [33]. The second step is associated with the rearrangement of the particles at the interface. This two-step scenario is in agreement with the numerical calculations by Colosqui et al. [107], who showed that the incorporation of particles to the fluid interface and the reorganization process occurring after their attachment are governed by an energy minimization principle. When surfactant decorated particles are considered, it is possible to attribute this process to 


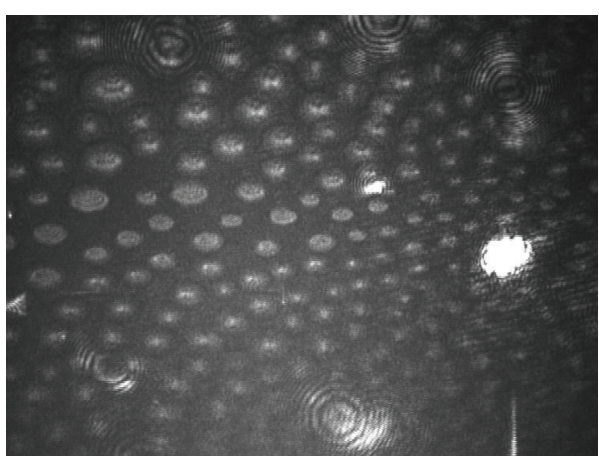

(a)

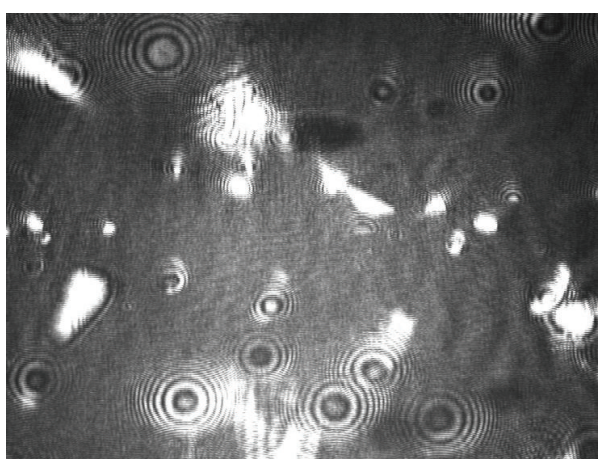

(c)

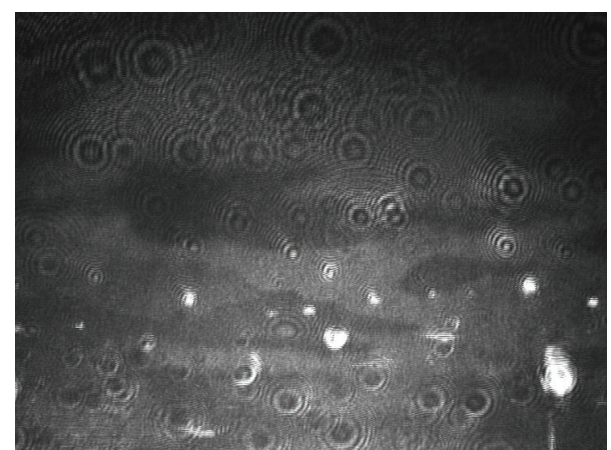

(b)

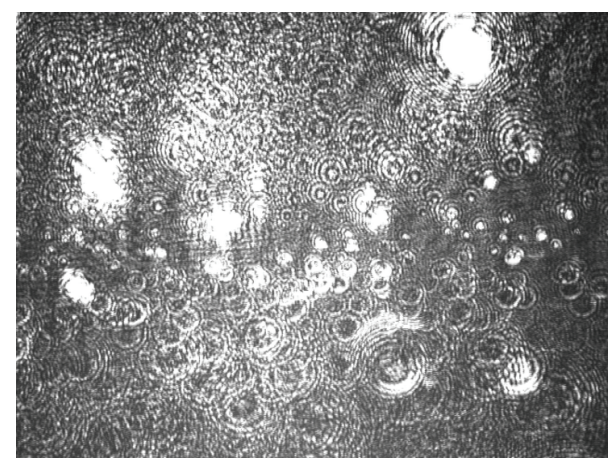

(d)

FIGURE 4: BAM images for PA-silica nanoparticles interfacial layers with different PA concentrations, namely, (a) $2 \times 10^{-5} \mathrm{M}$, (b) $5 \times 10^{-5} \mathrm{M}$, (c) $8 \times 10^{-5} \mathrm{M}$, and (d) $1 \times 10^{-4} \mathrm{M}$. Reprinted from [50]. Reproduced by permission of the PCCP Owner Societies.

the reorganization of surfactant molecules between the surface of the particles and the fluid interface, and it depends on the surfactant concentration. Indeed, the dynamic of the surfactants reorganization must be affected by the amount of surfactant transferred to the surface-carried by the particles-and by the area fraction occupied by the nanoparticles after their adsorption.

Two-step adsorption processes have been observed in the formation of interfacial layer by mixtures of silica nanoparticles and octadecylamine (ODA) at the water/hexane interface. The particularity of this systems is that the surfactant and the nanoparticles are dissolved/dispersed in a different phase and their interaction occurs only at the interface [38]. In this case, the first step with a characteristic time, around $10^{2} \mathrm{~s}$, is associated with the adsorption of the oil soluble surfactant, whereas the second step with a time span up $10^{4} \mathrm{~s}$ can be explained considering the interfacial interaction of silica nanoparticles and ODA. Thus, the second step can be assigned to the adsorption of particles to the preformed surfactant layer and the subsequent reorganization of the particle-laden interface. It is worth mentioning that the interfacial interaction between silica nanoparticles and ODA provoked a strong synergistic effect on the surface tension reduction, in a similar way to that found for PA-silica nanoparticles at the water/hexane interface [49].

The main parameter that governs the dynamics of particle incorporation into the interface is their hydrophilic/ hydrophobic character, so it is possible to analyze the dynamic of particle adsorption to the fluid interfaces in terms of the contact angle equilibration $[2,64]$. The formation of a three-phase contact line is expected to play a central role in the formation of particle-laden interface. This process is not continuous due to the rupture of the interface occurring by the particle contact. This process is characterized by different relaxation mechanisms till the particles reach their final position associated with a certain value of $\theta$. The dynamics of the wetting of particles has been recently evaluated by the using digital holographic microscopy $[64,107]$. For conditions in which the formation of the particle-laden interface is affected by strong electrostatic interaction, the formation of the contact line is not affected by any relaxation process and once the particles arrive to the interface they remain trapped at a fixed position. However, when the relaxation takes place, this is slower than expected considering only hydrodynamics interactions. The adsorption/spreading of the particles at the interface is a nonequilibrium process mediated by thermal fluctuations of the three-phase contact line around different positions separated by different activation energy barriers [108]. It is expected that the surface interactions occurring between the particles and the interface can affect the wetting dynamics [70]. It was found that the surface nature plays a key role in the wetting dynamic. Thus, it is possible to consider that the nanoscale surface features of the particles modify strongly the attachment of soft colloids to fluid interfaces. The relaxation processes are mainly controlled by molecular dissipation mechanism, being the role of the hydrodynamic 
interactions almost negligible. This allows considering the wetting dynamics of particles by fluid interfaces similar to that observed for physical aging processes of glassy materials [107].

It is worth mentioning that the dynamics of wetting is expected to change strongly from those particle-laden interfaces formed by direct assembly from the bulk dispersions and those formed by the spreading of the particles at the interface [39]. This is explained considering that, in the former case, the adsorption of the particles from fluid 1 to the interface implies the partial dewetting process of the particle from fluid 1 and the creation of the contact line. This process present a longer temporal scale than that expected for the thermal induced depinning of the formation of the contact line from the particle surface. On the other side, there are not effects of the temporal scale on the formation of particleladen interfaces by the direct spreading of the particles at the interface. In these cases, the deposition of the particles at the interface is a fast process in which turbulent flows of the solvent can occur at the interface. This phenomenon is also applied to the stabilization of emulsion where the mechanical energy is enough to overcome the depinning energy [71]. In this context, it is expected that the depinning energy can play a key role in the formation of particle-laden interfaces.

\section{Rheological Behavior of Particle-Laden Interfaces}

The understanding of the response of the particle-laden interfaces against mechanical deformation allows one to know the dynamics exchange and relaxation that leads to the particle to their steady state $[3,109,110]$. Additionally, the knowledge of the mechanical properties of particle-laden interfaces provides important insights for the development of technological applications in which the interaction of particles and interfaces is involved [3, 32] such as emulsification and foaming, phase transfer catalysis, encapsulation, or enhanced oil recovery [111]. It is worth mentioning that the interest of the mechanical response of particle-laden interface is related to its importance in many processes with a practical interest. The structural and morphological richness that presents particle-laden interfaces leads to an important variability of the rheological behaviour of these systems. This section will summarize the most important features related to the rheological behaviour of particle-laden interface against shear and dilational deformations.

5.1. Dilational Rheology. The dilational rheology provides information related to the changes of surface tension after the change of the area of the film; thus it can be expected that this type of changes affects the adsorption state of the particles and their structure due to the stress induced by the external deformation. The relaxation mechanisms observed under dilational deformation are strongly dependent on the time-scale studied $[53,112]$. The first studies were carried out by Miller et al. [98], who developed a theoretical model based on thermodynamic aspects associated with the adsorption isotherm developed of these systems [48]. This model was analogous to that previously developed for proteins and protein-surfactant layers [113]. The main novelty of the model was the introduction of a new parameter, the so-called cohesion pressure, that accounts for the cohesive interactions occurring between particles at the interfaces and that is strongly dependent on the wettability of the particles for the interface. Nevertheless the model has only tested data of a reduced number of systems.

It seems reasonable that the contact angle plays an important role in controlling the mechanical properties of particleladen interfaces because it controls its structure. Safouane et al. [114] analyzed the effect of the hydrophobicity of fumed silica nanoparticles on the rheological response of the particle-laden interface using capillary wave technique in the frequency range of 200-990 Hz. Their results showed a strong dependence of the dilational response on the hydrophobicity of the particles, which was associated with differences on the morphology of the particle-laden interfaces. In this case the elasticity, $E^{\prime}$, is around one order of magnitude larger than the viscous component, $E^{\prime \prime}$, independently of the hydrophobicity degree of the particles. However, the higher the hydrophobicity the higher the interfacial density and, consequently, the more rigid the interface. The increase in the particle hydrophobicity from total hydrophilic to total hydrophobic particles leads to the increase of the value of $E^{\prime}$ for a factor two, whereas $E^{\prime \prime}$ remains close to zero independently of the hydrophobicity degree of the particles. Zahn et al. [115] pointed out that using paramagnetic particles adsorbed at the air/water interface. The dilational response of layers formed by silica particles at the air/water interface presents a long linear regime [116, 117]. Zang et al. [51] studied the frequency dependence of silica particles layers at the air/water interface and did not find any relaxation process in the lowest frequency range studied $(0.016-0.1 \mathrm{~Hz})$. However, expanding the frequency range, they found a dynamic relaxation with a characteristic time close to $10^{3} \mathrm{~s}$. This process can be attributed to the internal reorganization of the layer, being faster as the particle hydrophobicity is reduced [118]. Zang et al. [119] extended the work of Safoune et al. [114] and found that $E^{\prime}$ increases with the hydrophobicity of the particles till a maximum value, which appears at intermediate values of the hydrophobicity.

The behavior of spread latex particles at the air/water interface is slightly different than the above discussed. In this case, the rheological response is strongly dependent on the region of the $2 \mathrm{D}$ phase diagram analyzed $[18,120] . E^{\prime}$ was found to be larger than $E^{\prime \prime}$ along the entire phase diagram of the system, and the behavior was independent of the strain rate for experiments performed at fixed frequencies [121]. A small hysteresis, associated with the loss connection between the particles, was found for these systems. The frequency dependence of $E^{\prime}$ and $E^{\prime \prime}$ pointed out a crossover between a fluid-like to a solid-like behavior. For low surface pressures, $E^{\prime}$ increases with the surface pressure due to repulsive electrostatic interaction between the particles. When the electrostatic repulsion is reduced by charge screening phenomena, both $E^{\prime}$ and $E^{\prime \prime}$ increase with the compression, till a threshold value of the surface pressure associated with 
the formation of a close packed monolayer, as was shown by using BAM images [120], with $E^{\prime}$ values in the range of $350-$ $600 \mathrm{mN} / \mathrm{m}$. Once the surface pressure threshold is overcome, both $E^{\prime}$ and $E^{\prime \prime}$ decrease with compression due to the collapse of the particle-laden layer and the appearance of buckling in the layer. It is worth mentioning that the particles monolayers can form dense monolayers even if the surface coverage remains relatively low [18], which frequently leads to small linear viscoelastic regime under dilational deformations [117].

All the above refer to interfaces filled exclusively by particles; however more attention has been paid to the dilational response of systems formed by particles and surfactant mixtures [1]. In the following we will discuss the rheology of this type of interfaces.

Ravera et al. [33] pointed out that the bulk interaction of hydrophilic silica nanoparticles and CTAB increased their hydrophobicity, thus leading to their adsorption at the interface. The rheological behavior of these monolayers in the low frequency range $(0.005-0.2 \mathrm{~Hz})$ was quite different than those of the pure surfactant. The effects of the particles were evidenced in both air/water and water/hexane interfaces. They explained these results in terms of the adsorption of particlesCTAB complexes with different degree of hydrophobicity, which lead to higher elasticity than for surfactant layers with similar surfactant concentration. Further studies showed that the [34] dilational rheology is strongly frequency dependent, with two relaxations at well-separated time scales. The low frequency process was associated with the diffusive transport of the particle to the interface, whereas the high frequency one was explained in terms of the exchange of surfactant molecules between the interface and the bulk since once the particles arrive to the interface it expected that they remain irreversibly attached. The analysis of the results in terms of a theoretical model accounting for the physical origin of the two processes evidenced a transition from a pure diffusive dynamics to a behavior mixing diffusion and reorganization of the layers as the hydrophobicity of the particles increased. A more detailed study of this type of systems with a larger frequency range (from $10^{-3}$ to $10^{2} \mathrm{~Hz}$ ) provided the bases for a more quantitative and qualitative analysis [37] of the kinetic aspects of particle-laden interfaces. Figure 5 shows the frequency dependence of $E^{\prime}$ and $E^{\prime \prime}$ for a broad range of frequencies of silica nanoparticles-CTAB monolayers and the corresponding fits using the theoretical model proposed by Ravera et al. [34]. Similar results were found by Wang et al. [122].

The study of the system formed by mixtures of silica nanoparticles and CTAB has pointed out the complex balance of interactions governing the rheological response of particle-laden interfaces $[52,53]$. Among these interactions the transitions occurring in the hydrophobicity degree of the particles by the attachment of surfactant molecules, the CTAB depletion from the bulk solution and the interactions between the particles in the bulk and at the interface are the most important.

Additional aspects to consider are the aging phenomenon of the interfacial layer $[34,39]$ that increases the rigidity at both air/water and water/hexane interface, although

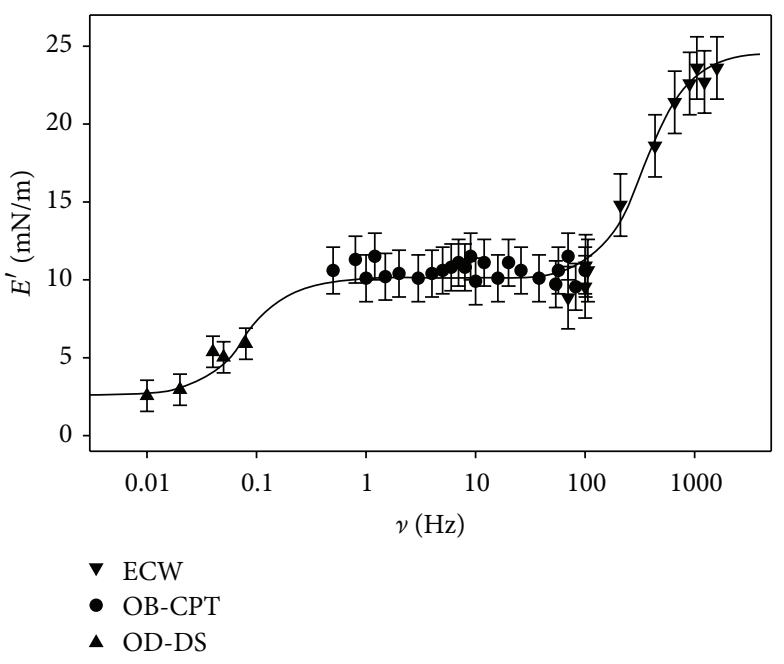

(a)

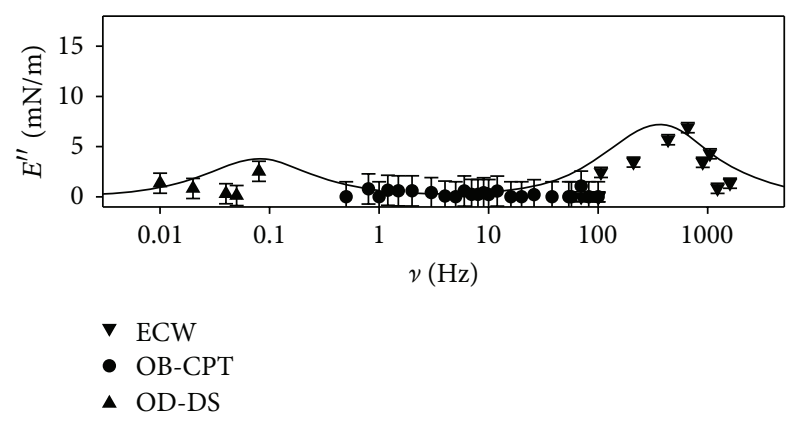

(b)

Figure 5: For dispersion with $1 \mathrm{wt} \%$ of silica nanoparticles and CTAB in concentration of $0.5 \mathrm{mM}$ : (a) Real part of the viscoelastic modulus. (b) Imaginary part of the viscoelastic modulus. The solids lines correspond to the best fitting to the model proposed by Ravera et al. [34]. Reproduced from [37] with permission from The Royal Society of Chemistry.

the surface tension of the particle-laden interface does not evidence any modification. This can be explained considering the irreversible attachment of the particles at the interface $[39,123]$ that forms a solid-like layers associated with a densification phenomenon as was evidenced by BAM [39].

5.2. Shear Rheology. Shear deformation accounts for shape deformations of the interface without modification of the interfacial area $[3,4]$. Intense theoretical and experimental efforts have been done to establish correlations between the shear responses and the morphology and potential applications of particle layers. It seems reasonable to expect that the monolayer structure will play a very important role in the control of the response of particle-laden interfaces against shear deformations [124]. The high interest of shear aspects in technological applications of particle-laden interfaces, especially in the stabilization of dispersed systems, has produced a higher development than in the case of the dilational counterpart. 
Cicuta et al. [125] performed one of the first studies dealing with the effect of the shear deformation of particleladen interfaces. They pointed out that, for polystyrene latex particles ( $3 \mu \mathrm{m}$ of diameter) monolayers at the water/decane, the viscous modulus $\left(G^{\prime \prime}\right)$ was larger than the elastic modulus $\left(G^{\prime}\right)$. Furthermore, this behaviour was rather independent of the deformation frequency. On the contrary, $\beta$-lactoglobulin layers present a mainly elastic behaviour. Thus, it is expected that the nondeformable characteristic of the particles can induce specific features on the rheological response. Additionally, Cicuta et al. [125] found a sharp increase of the viscoelastic modulus from values corresponding to the pure interface and reached a plateau for surface coverage around 0.75-0.80. This was associated with a jamming phenomenon occurring on the particle-laden interface. It is worth mentioning that the existence of both long-range and shortrange interactions in particle-laden interfaces introduces important differences between the behaviour of interfacial systems and their bulk counterparts [126]. The effect of the surface coverage on the response against shear of particleladen interfaces was discussed by Imperiali et al. [127], who showed that graphene oxide particles at fluid interfaces had a plastic-like behaviour below the close-packing, whereas for close-packed monolayers a perfect elastic behaviour was found. Such a transition was justified in terms of change of the free area available for the particles at the interface and consequently their mobility. The dependence of the shear response on the interfacial coverage has been also studied by Barman and Christopher [128], who found that shear thinning phenomena appeared at low surface coverage values, whereas yielding was found as the interfacial density increased. Thus, the differences found in the viscoelastic behaviour associated with interfacial reorganization of the particles can be rationalized considering different mechanisms of viscous dissipation occurring in the particle-laden interface. Furthermore, close-packed monolayers showed a nonlinear response against shear deformation.

The results mentioned above pointed out that the reorganization phenomena occurring in particle-laden interface are strongly correlated to the structural features of the monolayer. This was further studied by Reynaert et al. [129] who compared the response of particle-laden interfaces with different structures: 2D colloidal crystal and 2D aggregate suspensions. They found that aggregate layers showed a behaviour rather similar to that found for their bulk counterparts [126], being in agreement with the simulations performed by Wijmans and Dickinson [130]. 2D aggregate suspensions present a small linear regime with a power law dependence of the viscoelastic modulus on the surface coverage. Additionally, Reynaert et al. [129] confirmed the importance of the interparticle interaction in the control of the rheological response.

Another factor that affects the interfacial organization is the shape of the particles, and therefore it must also affect the shear response of the monolayers [131]. This was demonstrated by Madivala et al. [131] using ellipsoidal particles at the water/decane and water/air interfaces. Additionally, they found a low linearity range on the shear response, which decreases with the surface coverage. In the case of monolayers at the air/water interface, the shear elasticity is smaller than at water/decane interface. Ellipsoidal particles showed very high values of the elastic modulus even for low surface coverage values, whereas for spherical particles it remained close to that of the pure interface till reaching a threshold value of the surface coverage [125]. Furthermore, the values of the storage modulus found for ellipsoidal particles were almost one order of magnitude higher than those found for spherical particles at the same interface [129], which was attributed to the differences on the interfacial organization of the particles. Basavaraj et al. [132] pointed out that contrary to spherical particles particle-laden layers of ellipsoidal particles undergone buckling transitions for high values of the surface coverage, which can be understood when the higher complexity of the phase behaviour of nonspherical particles is considered. Brown et al. [133] studied analyzing four different types of nonspherical particles with different aspect ratio. They found that the coverage threshold for jamming was lower for nonspherical particles than for spherical ones. The value of this threshold decreases as the asymmetry of the particles increased. Additionally, depending on the particle symmetry, different shear flow patterns indicated arrested dynamic at the interface [133-135]. It was also that the charge density of the particles plays an important role [134]. Particle size also influences the shear response of particle monolayers [133] since it affects the phase diagram of the monolayer [18].

Particle roughness modifies the shear response of particle-laden interfaces [136] in such way that the increase of the particle roughness reduces the shear viscosity of the particle-laden interface in the low coverage region, the opposite being true for interfacial densities close to jamming [137]. This behaviour is attributed to differences on the interparticle friction occurring in the monolayer and is on the basis of the explanation of the shear thickening of particles monolayers [133].

Safouane et al. [114] using fumed silica particles at the air/ water interface found that particle wettability plays an important role in the rheological response of particle monolayers. Both $G^{\prime}$ and $G^{\prime \prime}$ increase with the particle hydrophobicity, reaching values around $10^{-1} \mathrm{mN} / \mathrm{m}$ that are several orders of magnitude larger than those found for pure surfactant layers (around $10^{-3} \mathrm{mN} / \mathrm{m}$ ). Particles with low hydrophobicity showed a mainly elastic behavior with a transition to viscous behaviour as the hydrophobicity of the particles increases, the crossover point (gel point, $G^{\prime}=G^{\prime \prime}$ ) being found for particles with the $36 \%$ of the silanol groups chemically silanized which present a contact angle around $90^{\circ}$. Zang et al. $[51,119,138]$ further studied the aforementioned layers, exploring the dependence of the shear modulus on the strain amplitude. They found that $G^{\prime}$ was rather independent of the strain amplitude for low values of the strain amplitude and found that $G^{\prime}>G^{\prime \prime}$ in the entire range of strain amplitude considered (several orders of magnitude). The melting of the monolayers was found above a threshold value of the strain amplitude (yield strain amplitudes) which is related to maximum value of $G^{\prime \prime}$. Once the yield amplitude is overcome a sudden drop of $G^{\prime}$ was found. For low deformation frequencies and at fixed strain, values of $G^{\prime \prime}$ larger than those of $G^{\prime}$ were found, whereas a crossover was found as the frequency 
increased that coincides with a maximum $G^{\prime \prime}$. This is due to a decrease of the structural relaxation time as the strain rate amplitude increases, similarly to that found in $3 \mathrm{D}$ soft solids [139]. The results pointed out that the relaxation shows a quasilinear dependence on the shear rate. An additional feature found for the particle-laden interfaces was the selfhealing character when the stress is released, which depends on the coverage of the interface and the wettability of the particles. This also affects the yield and melting stresses, which reach their maximum values for particles with contact angle around $90^{\circ}$. Similar behavior was found by Vandebril et al. [140].

Contrarily to what was found in dilational rheology studies, the studies of the response against shear of particlesurfactant systems are scarce. Maestro et al. [141] reported the effect of surfactant concentration in the rheological properties of CTAB decorated silica nanoparticles by oscillatory shear measurements. In all the samples studied, the interface is highly packed, with a remarkable solid-response against shear below a yield point. Maas et al. [142] studied the interfacial interaction of lipid and silica nanoparticles at the water/oil interface. They found a dynamic evolution of $G^{\prime}$ and $G^{\prime \prime}$ with time and found two well-differentiated behaviors as function of the time span consider. During the first stages of the adsorption process (first hour), $G^{\prime}$ increases till the formation of a rigid layer with $G^{\prime}>G^{\prime \prime}$, and at longer times $G^{\prime}$ increases more slowly, which was related to the accumulation of more particles at the interface. The increase of the moduli progresses until a steady state situation is reached with the shear response mainly governed by the dynamic exchange of material, probably surfactant molecules, between the bulk phases. Similar conclusions were obtained by Degen et al. $[143,144]$ in their studies of the interaction of $\gamma-\mathrm{Fe}_{2} \mathrm{O}_{3}$ and different surfactants.

\section{Particle Stabilized Disperse Systems: Foams, Emulsions, and Solid Foams}

The interfacial and mechanical properties of single interfaces are responsible for the formation and stabilization of the disperse systems (foams and emulsions) obtained with the same components. In fact, the properties of the liquid interface can hinder the processes which lead to foam/emulsion destabilization, such as creaming (or sedimentation), flocculation, coalescence, and Ostwald ripening $[145,146]$. The stabilization of the disperse systems is strictly related to the stability of the liquid film formed between two drops/bubbles approaching each other. This stabilization is related to the rate of film drainage from a thick to a thin film and from the other side to the stability of the final thin film to mechanical or thermal disturbance: both processes may strongly depend upon surfactant interfacial properties. One important aspect of the film stability concerns the role of the dilational rheological properties, which are strictly related to the dilational viscoelasticity of the single interfaces forming the film. These properties are in fact an expression of the capability of the layers to dampen the external disturbances, which could destabilize and eventually break the film.
The presence of nanoparticles at the fluid interfaces can play an important role in preventing the coalescence of drops and bubbles and, hence, in stabilising emulsions and foams. In these cases the emulsion morphology or the droplet size distribution is determined by the dimensions and the contact angles of particles at the liquid-liquid interface and, consequently, bubble or drop coalescence is prevented essentially by steric effect. An important role in the stabilization of the dispersed systems is played by the jamming of the particles layer as was pointed out by Stratford et al. [147] by largescale simulations. They showed that jammed particle layers can arrest the coalescence of two miscible fluid phases, thus allowing for an extrapolation of the physic principles involved in the stabilization of dispersed systems. The importance of the jamming in the stabilization of foams has been recently demonstrated by Maestro et al. [43].

Stancik et al. [135] and Horozov et al. [148] confirmed the role of the interfacial coverage in the stabilization of emulsions by nanoparticles. They pointed out that the role of the particle-laden interface in the stabilization of dispersed systems is not limited exclusively to the modification of the mechanical properties, being important the steric hindrance induced by the nanoparticles to prevent the coalescence. The effectiveness of colloidal particles in stabilizing emulsions depends in part on the formation of a sufficiently dense layer of particles at the interface. The rheological properties of the interfacial layers also change as the concentration of particles at the interface increases and complete surface coverage is achieved. For high particle concentration, the interfaces exhibit viscoelastic behaviour, the viscous properties being dominant at low concentrations, while the elastic properties are dominant at high particle concentrations. The elastic contribution to the viscoelastic behaviour is largely due to the interparticle interaction. The viscoelastic nature of the interfaces affects the emulsion stability by decreasing the rate of film thinning between coalescing droplets/bubbles.

The high stability of disperse systems in the presence of particles adsorbed at the fluid interface is a starting point for the preparation of tailored materials, such as solid foams. These porous materials can be obtained by drying and sintering the original wet foams/emulsions or by the solidification of the bulk liquid phase [10]. Zabiegaj et al. [104, 149] have investigated the relation between the interfacial properties of particle-laden interfaces and the stability/structure of the corresponding liquid and solid foams in the presence of carbon soot and alumina nanoparticles. They found out an enhanced stability of the wet foams when nanoparticles are added, mainly due to a steric effect. In fact, the formation of a second layer onto the drop surface acts as a steric barrier against the droplet collapse although any effect on the interfacial properties does occur.

\section{Concluding Remarks}

We consider that a key factor that is highlighted in this review is the versatility of particulate systems to control their morphology and strength when forming interfacial layers at fluid interfaces. This is a remarkable feature in 
order to create complex and hierarchical structures-from colloidosomes to foams and emulsions-that have many important industrial applications. The majority of work to date has reported the wettability of micro- and nanometer particle systems adsorbed at or spread to fluid interfaces through the measurement of their contact angle. Also, many efforts have been done to show the adsorption isotherm, as well as the equation of state for such particle-laden interfaces that correlates the surface tension with the density of particles at the interface. Looking forward, a major challenge is the lack of theoretical works to show a close agreement with the experimental studies and predict the behaviour of the particles at the interface. Multidisciplinary efforts will be required to fully understand the adsorption and final steady state morphology of the particle-laden interfaces. Up to date, interfacial rheological techniques provide a unique source of information about the intrinsic relation of the mechanical response and the interface morphology. We have emphasized in this review the importance of rheological techniques investigating both the dilatational and the shear modes of deformation, in order to understand the interfacial particle networks. The process of assembly that leads from amorphous interfaces, with only short range order, to crystalline structures, with a long range one, can be studied by rheological measurements. Also, interfacial rheology can help us to identify the instability mechanism that affects bubbles and droplets.

\section{Conflict of Interests}

The authors declare that the submitted paper does not present any conflict of interests.

\section{Acknowledgments}

This work was funded in part by MINECO under Grant FIS2012-38231-C02-01, by EU under Marie Curie ITN CoWet, and by ESA under Grant PASTA and performed in the framework of COST Actions CM-1101 and MP-1106. Eduardo Guzman and Armando Maestro are grateful to the MINECO for the JdC contract and to the RSC for the Newton contract, respectively. They are grateful to C.A.I. Espectroscopía Raman-Correlación-FT-IR (UCM) for the use of their facilities.

\section{References}

[1] E. Guzmán, E. Santini, L. Liggieri et al., "Particle-surfactant interaction at liquid interfaces," in Colloid and Interface Chemistry for Nanotechnology, P. Kralchevsky, R. Miller, and F. Ravera, Eds., pp. 77-109, CRC Press, Taylor \& Francis, Boca Raton, Fla, USA, 2013.

[2] A. Maestro, E. Guzmán, F. Ortega, and R. G. Rubio, "Contact angle of micro- and nanoparticles at fluid interfaces," Current Opinion in Colloid \& Interface Science, vol. 19, no. 4, pp. 355367, 2014.

[3] A. J. Mendoza, E. Guzmán, F. Martínez-Pedrero et al., "Particle laden fluid interfaces: dynamics and interfacial rheology,"
Advances in Colloid and Interface Science, vol. 206, pp. 303-319, 2014.

[4] B. P. Binks and T. S. Horozov, Colloidal Particles at Liquid Interfaces, Cambridge University Press, Cambridge, UK, 2006.

[5] R. Aveyard, B. P. Binks, and J. H. Clint, "Emulsions stabilised solely by colloidal particles," Advances in Colloid and Interface Science, vol. 100-102, pp. 503-546, 2003.

[6] B. P. Binks, "Particles as surfactants-similarities and differences," Current Opinion in Colloid and Interface Science, vol. 7, no. 1-2, pp. 21-41, 2002.

[7] A. D. Dinsmore, M. F. Hsu, M. G. Nikolaides, M. Marquez, A. R. Bausch, and D. A. Weitz, "Colloidosomes: selectively permeable capsules composed of colloidal particles," Science, vol. 298, no. 5595, pp. 1006-1009, 2002.

[8] O. D. Velev and S. Gupta, "Materials fabricated by micro- and nanoparticle assembly-the challenging path from science to engineering," Advanced Materials, vol. 21, no. 19, pp. 1897-1905, 2009.

[9] H. Zhang and A. I. Cooper, "Synthesis and applications of emulsion-templated porous materials," Soft Matter, vol. 1, no. 2, pp. 107-113, 2005.

[10] A. R. Studart, U. T. Gonzenbach, I. Akartuna, E. Tervoort, and L. J. Gauckler, "Materials from foams and emulsions stabilized by colloidal particles," Journal of Materials Chemistry, vol. 17, no. 31, pp. 3283-3289, 2007.

[11] E. Dickinson, "Food emulsions and foams: stabilization by particles," Current Opinion in Colloid and Interface Science, vol. 15, no. 1-2, pp. 40-49, 2010.

[12] N. Thongprachan, T. Yamamoto, J. Chaichanawong, T. Ohmori, and A. Endo, "Preparation of macroporous carbon foam using emulsion templating method," Adsorption, vol.17, no. 1, pp. 205210, 2011.

[13] T. N. Hunter, R. J. Pugh, G. V. Franks, and G. J. Jameson, “The role of particles in stabilising foams and emulsions," Advances in Colloid and Interface Science, vol. 137, no. 2, pp. 57-81, 2008.

[14] J. Ralston, D. Fornasiero, and R. Hayes, "Bubble-particle attachment and detachment in flotation," International Journal of Mineral Processing, vol. 56, no. 1-4, pp. 133-164, 1999.

[15] D. I. Verrelli, P. T. L. Koh, and A. V. Nguyen, "Particle-bubble interaction and attachment in flotation," Chemical Engineering Science, vol. 66, no. 23, pp. 5910-5921, 2011.

[16] P. B. Kowalczuk and J. Drzymala, "Surface flotation of particles on liquids. Principles and applications," Colloids and Surfaces A: Physicochemical and Engineering Aspects, vol. 393, pp. 81-85, 2012.

[17] I. Blute, R. J. Pugh, J. van der Pas, and I. Callaghan, "Silica nanoparticle sols: 1 . Surface chemical characterization and evaluation of the foam generation (foamability)," Journal of Colloid \& Interface Science, vol. 313, no. 2, pp. 645-655, 2007.

[18] L. J. Bonales, J. E. F. Rubio, H. Ritacco, C. Vega, R. G. Rubio, and F. Ortega, "Freezing transition and interaction potential in monolayers of microparticles at fluid interfaces," Langmuir, vol. 27, no. 7, pp. 3391-3400, 2011.

[19] L. J. Bonales, F. Martínez-Pedrero, M. A. Rubio, R. G. Rubio, and F. Ortega, "Phase behavior of dense colloidal binary monolayers," Langmuir, vol. 28, no. 48, pp. 16555-16566, 2012.

[20] E. Santini, F. Ravera, M. Ferrari, M. Alfè, A. Ciajolo, and L. Liggieri, "Interfacial properties of carbon particulate-laden liquid interfaces and stability of related foams and emulsions," Colloids and Surfaces A: Physicochemical and Engineering Aspects, vol. 365, no. 1-3, pp. 189-198, 2010. 
[21] E. Santini, E. Guzmán, F. Ravera et al., "Soot particles at the aqueous interface and effects on foams stability," Colloids and Surfaces A: Physicochemical and Engineering Aspects, vol. 413, pp. 216-223, 2012.

[22] E. Guzmán, E. Santini, A. Benedetti, F. Ravera, M. Ferrari, and L. Liggieri, "Surfactant induced complex formation and their effects on the interfacial properties of seawater," Colloids and Surfaces B: Biointerfaces, vol. 123, pp. 701-709, 2014.

[23] E. Guzmán, L. Liggieri, E. Santini, M. Ferrari, and F. Ravera, "Effect of hydrophilic and hydrophobic nanoparticles on the surface pressure response of DPPC monolayers," Journal of Physical Chemistry C, vol. 115, no. 44, pp. 21715-21722, 2011.

[24] E. Guzmán, L. Liggieri, E. Santini, M. Ferrari, and F. Ravera, "Influence of silica nanoparticles on dilational rheology of DPPC-palmitic acid Langmuir monolayers," Soft Matter, vol. 8, no. 14, pp. 3938-3948, 2012.

[25] E. Guzmán, L. Liggieri, E. Santini, M. Ferrari, and F. Ravera, "DPPC-DOPC langmuir monolayers modified by hydrophilic silica nanoparticles: phase behaviour, structure and rheology," Colloids and Surfaces A: Physicochemical and Engineering Aspects, vol. 413, pp. 174-183, 2012.

[26] E. Guzmán, L. Liggieri, E. Santini, M. Ferrari, and F. Ravera, "Influence of silica nanoparticles on phase behavior and structural properties of DPPC-Palmitic acid Langmuir monolayers," Colloids and Surfaces A: Physicochemical and Engineering Aspects, vol. 413, pp. 280-287, 2012.

[27] E. Guzmán, L. Liggieri, E. Santini, M. Ferrari, and F. Ravera, "Mixed DPPC_cholesterol Langmuir monolayers in presence of hydrophilic silica nanoparticles," Colloids and Surfaces B: Biointerfaces, vol. 105, pp. 284-293, 2013.

[28] E. Guzmán, D. Orsi, L. Cristofolini, L. Liggieri, and F. Ravera, "2D DPPC based emulsion-like structures stabilized by silica nanoparticles," Langmuir, vol. 30, no. 39, pp. 11504-11512, 2014.

[29] A. Moskal, T. R. Sosnowski, and L. Gradon, "Inhalation and deposition of nanoparticles: fundamentals, phenomenology and practical aspects," in Nanoparticles in Medicine and Environment, J. C. M. Marijnissen and L. Gradon, Eds., pp. 113-144, Springer Science+Business Media, Berlin, Germany, 2011.

[30] K. Kramek-Romanowska, M. Odziomek, and T. R. Sosnowski, "Dynamic tensiometry studies on interactions of novel therapeutic inhalable powders with model pulmonary surfactant at the air-water interface," Colloids and Surfaces A: Physicochemical and Engineering Aspects, vol. 480, pp. 149-158, 2015.

[31] P. Pieranski, "Two-dimensional interfacial colloidal crystals," Physical Review Letters, vol. 45, no. 7, pp. 569-572, 1980.

[32] V. Garbin, J. C. Crocker, and K. J. Stebe, "Nanoparticles at fluid interfaces: exploiting capping ligands to control adsorption, stability and dynamics," Journal of Colloid and Interface Science, vol. 387, no. 1, pp. 1-11, 2012.

[33] F. Ravera, E. Santini, G. Loglio, M. Ferrari, and L. Liggieri, "Effect of nanoparticles on the interfacial properties of liquid/liquid and liquid/air surface layers," Journal of Physical Chemistry B, vol. 110, no. 39, pp. 19543-19551, 2006.

[34] F. Ravera, M. Ferrari, L. Liggieri, G. Loglio, E. Santini, and A. Zanobini, "Liquid-liquid interfacial properties of mixed nanoparticle-surfactant systems," Colloids and Surfaces A: Physicochemical and Engineering Aspects, vol. 323, no. 1-3, pp. 99-108, 2008.

[35] W. Ramsden, "Separation of solids in the surface-layer of solutions and 'suspension' (observations on surface-membranes, bubbles, emulsions, and mechanical coagulation) - preliminary account," Proceedings of the Royal Society of London, vol. 72, pp. 156-164, 1903.
[36] S. U. Pickering, "Emulsions," Journal of the Chemical Society, Transactions, vol. 91, pp. 2001-2021, 1907.

[37] L. Liggieri, E. Santini, E. Guzmán, A. Maestro, and F. Ravera, "Wide-frequency dilational rheology investigation of mixed silica nanoparticle-CTAB interfacial layers," Soft Matter, vol. 7, no. 17, pp. 7699-7709, 2011.

[38] C. P. Whitby, D. Fornasiero, J. Ralston, L. Liggieri, and F. Ravera, "Properties of fatty amine-silica nanoparticle interfacial layers at the hexane-water interface," Journal of Physical Chemistry C, vol. 116, no. 4, pp. 3050-3058, 2012.

[39] P. A. Yazhgur, B. A. Noskov, L. Liggieri et al., "Dynamic properties of mixed nanoparticle/surfactant adsorption layers," Soft Matter, vol. 9, no. 12, pp. 3305-3314, 2013.

[40] A. C. Martinez, E. Rio, G. Delon, A. Saint-Jalmes, D. Langevin, and B. P. Binks, "On the origin of the remarkable stability of aqueous foams stabilised by nanoparticles: link with microscopic surface properties," Soft Matter, vol. 4, no. 7, pp. 15311535, 2008.

[41] A. Stocco, W. Drenckhan, E. Rio, D. Langevin, and B. P. Binks, "Particle-stabilised foams: an interfacial study," Soft Matter, vol. 5, no. 11, pp. 2215-2222, 2009.

[42] A. Stocco, E. Rio, B. P. Binks, and D. Langevin, "Aqueous foams stabilized solely by particles," Soft Matter, vol. 7, no. 4, pp. 12601267, 2011.

[43] A. Maestro, E. Rio, W. Drenckhan, D. Langevin, and A. Salonen, "Foams stabilised by mixtures of nanoparticles and oppositely charged surfactants: relationship between bubble shrinkage and foam coarsening," Soft Matter, vol. 10, no. 36, pp. 6975-6983, 2014.

[44] D. Orsi, G. Baldi, P. Cicuta, and L. Cristofolini, “On the relation between hierarchical morphology and mechanical properties of a colloidal 2D gel system," Colloids and Surfaces A: Physicochemical and Engineering Aspects, vol. 413, pp. 71-77, 2012.

[45] D. Orsi, L. Cristofolini, G. Baldi, and A. Madsen, "Heterogeneous and anisotropic dynamics of a 2D Gel," Physical Review Letters, vol. 108, no. 10, Article ID 105701, 2012.

[46] P. Kralchevsky and K. Nagayama, Eds., Particles at Fluid Interfaces and Membranes, Elsevier Science, New York, NY, USA, 2001.

[47] F. Bresme and M. Oettel, "Nanoparticles at fluid interfaces," Journal of Physics Condensed Matter, vol. 19, no. 41, Article ID 413101, 2007.

[48] V. B. Fainerman, V. I. Kovalchuk, E. H. Lucassen-Reynders et al., "Surface-pressure isotherms of monolayers formed by microsize and nanosize particles," Langmuir, vol. 22, no. 4, pp. 1701-1705, 2006.

[49] E. Santini, E. Guzmán, M. Ferrari, and L. Liggieri, "Emulsions stabilized by the interaction of silica nanoparticles and palmitic acid at the water-hexane interface," Colloids and Surfaces A: Physicochemical and Engineering Aspects, 2014.

[50] E. Santini, E. Guzmán, F. Ravera, M. Ferrari, and L. Liggieri, "Properties and structure of interfacial layers formed by hydrophilic silica dispersions and palmitic acid," Physical Chemistry Chemical Physics, vol. 14, no. 2, pp. 607-615, 2012.

[51] D. Y. Zang, E. Rio, D. Langevin, B. Wei, and B. P. Binks, "Viscoelastic properties of silica nanoparticle monolayers at the air-water interface," European Physical Journal E, vol. 31, no. 2, pp. 125-134, 2010.

[52] E. Santini, J. Krägel, F. Ravera, L. Liggieri, and R. Miller, "Study of the monolayer structure and wettability properties of silica nanoparticles and CTAB using the Langmuir trough 
technique," Colloids and Surfaces A: Physicochemical and Engineering Aspects, vol. 382, no. 1-3, pp. 186-191, 2011.

[53] A. Maestro, E. Guzmán, E. Santini et al., "Wettability of silica nanoparticle-surfactant nanocomposite interfacial layers," Soft Matter, vol. 8, no. 3, pp. 837-843, 2012.

[54] F. Ravera, M. Ferrari, L. Liggieri, R. Miller, and A. Passerone, "Measurement of the partition coefficient of surfactants in water/oil systems," Langmuir, vol. 13, no. 18, pp. 4817-4820, 1997.

[55] L. Isa, F. Lucas, R. Wepf, and E. Reimhult, "Measuring singlenanoparticle wetting properties by freeze-fracture shadowcasting cryo-scanning electron microscopy," Nature Communications, vol. 2, article 438, 2011.

[56] S. P. McBride and B. M. Law, "Influence of line tension on spherical colloidal particles at liquid-vapor interfaces," Physical Review Letters, vol. 109, no. 19, Article ID 196101, 2012.

[57] L. D. Landau, Fluid Mechanics, Pergamon Press, London, UK, 1959.

[58] A. W. Neumann, R. David, and Y. Zuo, Applied Surface Thermodynamics, CRC Press, Taylor \& Francis, Boca Raton, Fla, USA, 1996.

[59] I. B. Ivanov, P. A. Kralchevsky, and A. D. Nikolov, "Film and line tension effects on the attachment of particles to an interface. I. Conditions for mechanical equilibrium of fluid and solid particles at a fluid interface," Journal of Colloid \& Interface Science, vol. 112, no. 1, pp. 97-107, 1986.

[60] R. Aveyard and J. H. Clint, "Particle wettability and line tension," Journal of the Chemical Society_Faraday Transactions, vol. 92, no. 1, pp. 85-89, 1996.

[61] F. Bresme and N. Quirke, "Computer simulation study of the wetting behavior and line tensions of nanometer size particulates at a liquid-vapor interface," Physical Review Letters, vol. 80, no. 17, pp. 3791-3794, 1998.

[62] M. Zeng, J. Mi, and C. Zhong, "Wetting behavior of spherical nanoparticles at a vapor-liquid interface: a density functional theory study," Physical Chemistry Chemical Physics, vol. 13, no. 9, pp. 3932-3941, 2011.

[63] H. S. Wi, S. Cingarapu, K. J. Klabunde, and B. M. Law, "Nanoparticle adsorption at liquid-vapor surfaces: influence of nanoparticle thermodynamics, wettability, and line tension," Langmuir, vol. 27, no. 16, pp. 9979-9984, 2011.

[64] D. M. Kaz, R. McGorty, M. Mani, M. P. Brenner, and V. N. Manoharan, "Physical ageing of the contact line on colloidal particles at liquid interfaces," Nature Materials, vol. 11, no. 2, pp. 138-142, 2012.

[65] Y. Guo, D. Tang, Y. Du, and B. Liu, "Controlled fabrication of hexagonally close-packed langmuir-blodgett silica particulate monolayers from binary surfactant and solvent systems," Langmuir, vol. 29, no. 9, pp. 2849-2858, 2013.

[66] P. M. Hansson, L. Skedung, P. M. Claesson et al., "Robust hydrophobic surfaces displaying different surface roughness scales while maintaining the same wettability," Langmuir, vol. 27, no. 13, pp. 8153-8159, 2011.

[67] A. Maestro, L. J. Bonales, H. Ritacco, R. G. Rubio, and F. Ortega, "Effect of the spreading solvent on the three-phase contact angle of microparticles attached at fluid interfaces," Physical Chemistry Chemical Physics, vol. 12, no. 42, pp. 14115-14120, 2010.

[68] J. H. Clint and S. E. Taylor, "Particle size and interparticle forces of overbased detergents: a Langmuir trough study," Colloids and Surfaces, vol. 65, no. 1, pp. 61-67, 1992.

[69] V. N. Paunov, B. P. Binks, and N. P. Ashby, "Adsorption of charged colloid particles to charged liquid surfaces," Langmuir, vol. 18, no. 18, pp. 6946-6955, 2002.
[70] Z. Hórvölgyi, M. Máté, A. Dániel, and J. Szalma, "Wetting behaviour of silanized glass microspheres at water-air interfaces: a Wilhelmy film balance study," Colloids and Surfaces A: Physicochemical and Engineering Aspects, vol. 156, no. 1-3, pp. 501-507, 1999.

[71] L. Galet, S. Patry, and J. Dodds, "Determination of the wettability of powders by the Washburn capillary rise method with bed preparation by a centrifugal packing technique," Journal of Colloid and Interface Science, vol. 346, no. 2, pp. 470-475, 2010.

[72] B. Wei, Q. Chang, and C. Yan, "Wettability determined by capillary rise with pressure increase and hydrostatic effects," Journal of Colloid and Interface Science, vol. 376, no. 1, pp. 307311, 2012.

[73] A. Depalo and A. C. Santomaso, "Wetting dynamics and contact angles of powders studied through capillary rise experiments," Colloids and Surfaces A: Physicochemical and Engineering Aspects, vol. 436, pp. 371-379, 2013.

[74] G. Gillies, K. Büscher, M. Preuss, M. Kappl, H.-J. Butt, and K. Graf, "Contact angles and wetting behaviour of single micronsized particles," Journal of Physics: Condensed Matter, vol. 17, no. 9, pp. S445-S464, 2005.

[75] M. Preuss and H.-J. Butt, "Measuring the contact angle of individual colloidal particles," Journal of Colloid and Interface Science, vol. 208, no. 2, pp. 468-477, 1998.

[76] V. N. Paunov, "Novel method for determining the three-phase contact angle of colloid particles adsorbed at air-water and oilwater interfaces," Langmuir, vol. 19, no. 19, pp. 7970-7976, 2003.

[77] L. N. Arnaudov, O. J. Cayre, M. A. Cohen Stuart, S. D. Stoyanov, and V. N. Paunov, "Measuring the three-phase contact angle of nanoparticles at fluid interfaces," Physical Chemistry Chemical Physics, vol. 12, no. 2, pp. 328-331, 2010.

[78] B. P. Binks, L. Isa, and A. T. Tyowua, "Direct measurement of contact angles of silica particles in relation to double inversion of pickering emulsions," Langmuir, vol. 29, no. 16, pp. 49234927, 2013.

[79] J. S. Sander, L. Isa, P. A. Rühs, P. Fischer, and A. R. Studart, "Stabilization mechanism of double emulsions made by microfluidics," Soft Matter, vol. 8, no. 45, pp. 11471-11477, 2012.

[80] K. Geisel, L. Isa, and W. Richtering, "Unraveling the 3D localization and deformation of responsive microgels at oil/water interfaces: a step forward in understanding soft emulsion stabilizers," Langmuir, vol. 28, no. 45, pp. 15770-15776, 2012.

[81] D. O. Grigoriev, J. Krägel, V. Dutschk, R. Miller, and H. Möhwald, "Contact angle determination of micro- and nanoparticles at fluid/fluid interfaces: the excluded area concept," Physical Chemistry Chemical Physics, vol. 9, no. 48, pp. 6447-6454, 2007.

[82] D. O. Grigoriev, H. Möhwald, and D. G. Shchukin, “Theoretical evaluation of nano- or microparticulate contact angle at fluid/fluid interfaces: analysis of the excluded area behavior upon compression," Physical Chemistry Chemical Physics, vol. 10, no. 14, pp. 1975-1982, 2008.

[83] T. S. Horozov, D. A. Braz, P. D. I. Fletcher, B. P. Binks, and J. H. Clint, "Novel film-calliper method of measuring the contact angle of colloidal particles at liquid interfaces," Langmuir, vol. 24, no. 5, pp. 1678-1681, 2008.

[84] T. N. Hunter, G. J. Jameson, and E. J. Wanless, "Determination of contact angles of nanosized silica particles by multi-angle single-wavelength ellipsometry," Australian Journal of Chemistry, vol. 60, no. 9, pp. 651-655, 2007.

[85] T. N. Hunter, G. J. Jameson, E. J. Wanless, D. Dupin, and S. P. Armes, "Adsorption of submicrometer-sized cationic sterically 
stabilized polystyrene latex at the air-water interface: contact angle determination by ellipsometry," Langmuir, vol. 25, no. 6 , pp. 3440-3449, 2009.

[86] D. Zang, A. Stocco, D. Langevin, B. Wei, and B. P. Binks, "An ellipsometry study of silica nanoparticle layers at the water surface," Physical Chemistry Chemical Physics, vol. 11, no. 41, pp. 9522-9529, 2009.

[87] H. G. Tompkins, A User's Guide to Ellipsometry, Academic Press, Amsterdam, The Netherlands, 1993.

[88] A. Stocco, G. Su, M. Nobili, M. In, and D. Wang, "In situ assessment of the contact angles of nanoparticles adsorbed at fluid interfaces by multiple angle of incidence ellipsometry," Soft Matter, vol. 10, no. 36, pp. 6999-7007, 2014.

[89] J. Reguera, E. Ponomarev, T. Geue, F. Stellacci, F. Bresme, and M. Moglianetti, "Contact angle and adsorption energies of nanoparticles at the air-liquid interface determined by neutron reflectivity and molecular dynamics," Nanoscale, vol. 7, no. 13, pp. 5665-5673, 2015.

[90] M. A. Fernandez-Rodriguez, Y. Song, M. Á. RodríguezValverde, S. Chen, M. A. Cabrerizo-Vilchez, and R. HidalgoAlvarez, "Comparison of the interfacial activity between homogeneous and janus gold nanoparticles by pendant drop tensiometry," Langmuir, vol. 30, no. 7, pp. 1799-1804, 2014.

[91] M. A. Fernández-Rodríguez, M. A. Rodríguez-Valverde, M. Cabrerizo-Vílchez, and R. Hidalgo-Alvarez, "Surface activity and collective behaviour of colloidally stable Janus-like particles at the air-water interface," Soft Matter, vol. 10, no. 19, pp. 34713476, 2014.

[92] M. P. Boneva, N. C. Christov, K. D. Danov, and P. A. Kralchevsky, "Effect of electric-field-induced capillary attraction on the motion of particles at an oil-water interface," Physical Chemistry Chemical Physics, vol. 9, no. 48, pp. 6371-6384, 2007.

[93] B. J. Park, J. P. Pantina, E. M. Furst, M. Oettel, S. Reynaert, and J. Vermant, "Direct measurements of the effects of salt and surfactant on interaction forces between colloidal particles at water-oil interfaces," Langmuir, vol. 24, no. 5, pp. 1686-1694, 2008.

[94] K. M. Reed, J. Borovicka, T. S. Horozov et al., "Adsorption of sterically stabilized latex particles at liquid surfaces: effects of steric stabilizer surface coverage, particle size, and chain length on particle wettability," Langmuir, vol. 28, no. 18, pp. 7291-7298, 2012.

[95] Y. Nonomura and S. Komura, "Surface activity of solid particles with extremely rough surfaces," Journal of Colloid and Interface Science, vol. 317, no. 2, pp. 501-506, 2008.

[96] S. Levine, B. D. Bowen, and S. J. Partridge, "Stabilization of emulsions by fine particles I. Partitioning of particles between continuous phase and oil/water interface," Colloids and Surfaces, vol. 38, no. 2, pp. 325-343, 1989.

[97] A. W. Adamson and A. P. Gast, Physical Chemistry of Surfaces, John Wiley \& Sons, Boston, Mass, USA, 1997.

[98] R. Miller, V. B. Fainerman, V. I. Kovalchuk, D. O. Grigoriev, M. E. Leser, and M. Michel, "Composite interfacial layers containing micro-size and nano-size particles," Advances in Colloid and Interface Science, vol. 128-130, pp. 17-26, 2006.

[99] V. B. Fainerman and R. Miller, "Equation of state for concentrated protein surface layers at the water/air interface," Langmuir, vol. 15, no. 5, pp. 1812-1816, 1999.

[100] D. Zabiegaj, E. Santini, E. Guzmán, M. Ferrari, L. Liggieri, and F. Ravera, "Carbon soot-ionic surfactant mixed layers at water/air interfaces," Journal of Nanoscience and Nanotechnology, vol. 15, no. 5, pp. 3618-3625, 2015.
[101] L. R. Arriaga, W. Drenckhan, A. Salonen et al., "On the longterm stability of foams stabilised by mixtures of nano-particles and oppositely charged short chain surfactants," Soft Matter, vol. 8, no. 43, pp. 11085-11097, 2012.

[102] S. Llamas, E. Guzmán, F. Ortega et al., "Adsorption of polyelectrolytes and polyelectrolytes-surfactant mixtures at surfaces: a physico-chemical approach to a cosmetic challenge," Advances in Colloid and Interface Science, vol. 222, pp. 461-487, 2015.

[103] V. B. Fainerman, E. H. Lucassen-Reynders, and R. Miller, "Adsorption of surfactants and proteins at fluid interfaces," Colloids and Surfaces A: Physicochemical and Engineering Aspects, vol. 143, no. 2-3, pp. 141-165, 1998.

[104] D. Zabiegaj, E. Santini, M. Ferrari, L. Liggieri, and F. Ravera, "Carbon based porous materials from particle stabilized wet foams," Colloids and Surfaces A: Physicochemical and Engineering Aspects, vol. 473, pp. 24-31, 2015.

[105] C. Stefaniu, M. Chanana, D. Wang, D. V. Novikov, G. Brezesinski, and H. Möhwald, "Biocompatible magnetite nanoparticles trapped at the air/water interface," ChemPhysChem, vol. 11, no. 17, pp. 3585-3588, 2010.

[106] A. Stocco, J. Crassous, A. Salonen, A. Saint-Jalmes, and D. Langevin, "Two-mode dynamics in dispersed systems: the case of particle-stabilized foams studied by diffusing wave spectroscopy," Physical Chemistry Chemical Physics, vol. 13, no. 8, pp. 3064-3072, 2011.

[107] C. E. Colosqui, J. F. Morris, and J. Koplik, "Colloidal adsorption at fluid interfaces: regime crossover from fast relaxation to physical aging," Physical Review Letters, vol. 111, no. 2, Article ID 028302, 2013.

[108] G. L. Hunter and E. R. Weeks, "The physics of the colloidal glass transition," Reports on Progress in Physics, vol. 75, no. 6, Article ID 066501, 2012.

[109] R. Miller and L. Liggieri, Interfacial Rheology, Brill Publishers, Leiden, The Netherlands, 2009.

[110] L. Liggieri and R. Miller, "Relaxation of surfactants adsorption layers at liquid interfaces," Current Opinion in Colloid and Interface Science, vol. 15, no. 4, pp. 256-263, 2010.

[111] S. Crossley, J. Faria, M. Shen, and D. E. Resasco, "Solid nanoparticles that catalyze biofuel upgrade reactions at the water/oil interface," Science, vol. 327, no. 5961, pp. 68-72, 2010.

[112] A. Maestro, E. Guzmán, R. Chuliá, F. Ortega, R. G. Rubio, and R. Miller, "Fluid to soft-glass transition in a quasi-2D system: thermodynamic and rheological evidences for a Langmuir monolayer," Physical Chemistry Chemical Physics, vol. 13, no. 20, pp. 9534-9539, 2011.

[113] R. Miller, V. B. Fainerman, A. V. Makievski et al., "Dynamics of protein and mixed protein/surfactant adsorption layers at the water/fluid interface," Advances in Colloid and Interface Science, vol. 86, no. 1, pp. 39-82, 2000.

[114] M. Safouane, D. Langevin, and B. P. Binks, "Effect of particle hydrophobicity on the properties of silica particle layers at the air-water interface," Langmuir, vol. 23, no. 23, pp. 11546-11553, 2007.

[115] K. Zahn, A. Wille, G. Maret, S. Sengupta, and P. Nielaba, "Elastic properties of 2D colloidal crystals from video microscopy," Physical Review Letters, vol. 90, no. 15, Article ID 155506, 2003.

[116] H. Hilles, A. Maestro, F. Monroy, F. Ortega, R. G. Rubio, and M. G. Velarde, "Polymer monolayers with a small viscoelastic linear regime: equilibrium and rheology of poly(octadecyl acrylate) and poly(vinyl stearate)," The Journal of Chemical Physics, vol. 126, no. 12, Article ID 124904, 2007. 
[117] H. Hilles, F. Monroy, L. J. Bonales, F. Ortega, and R. G. Rubio, "Fourier-transform rheology of polymer Langmuir monolayers: analysis of the non-linear and plastic behaviors," Advances in Colloid and Interface Science, vol. 122, no. 1-3, pp. 67-77, 2006.

[118] D. Y. Zang, Y. J. Zhang, and Q. W. Hou, "Effect of hydrophobicity on tensile rheological properties of silica nanoparticle monolayers at the air-water interface," Colloids and Surfaces A: Physicochemical and Engineering Aspects, vol. 395, pp. 262-266, 2012.

[119] D. Y. Zang, E. Rio, G. Delon, D. Langevin, B. Wei, and B. P. Binks, "Influence of the contact angle of silica nanoparticles at the air-water interface on the mechanical properties of the layers composed of these particles," Molecular Physics, vol. 109, no. 7-10, pp. 1057-1066, 2011.

[120] A. G. Bykov, B. A. Noskov, G. Loglio, V. V. Lyadinskaya, and R. Miller, "Dilational surface elasticity of spread monolayers of polystyrene microparticles," Soft Matter, vol. 10, no. 34, pp. 6499-6505, 2014.

[121] T. Kobayashi and M. Kawaguchi, "Surface dilational moduli of latex-particle monolayers spread at air-water interface," Journal of Colloid and Interface Science, vol. 390, no. 1, pp. 147-150, 2013.

[122] H. Wang, Y. Gong, W. Lu, and B. Chen, "Influence of nano$\mathrm{SiO}_{2}$ on dilational viscoelasticity of liquid/air interface of cetyltrimethyl ammonium bromide," Applied Surface Science, vol. 254, no. 11, pp. 3380-3384, 2008.

[123] B. A. Noskov, P. A. Yazhgur, L. Liggieri et al., "Dilational rheology of spread and adsorbed layers of silica nanoparticles at the liquid-gas interface," Colloid Journal, vol. 76, no. 2, pp. 127-138, 2014.

[124] J. J. Gray and R. T. Bonnecaze, "Rheology and dynamics of sheared arrays of colloidal particles," Journal of Rheology, vol. 42, no. 5, pp. 1121-1151, 1998.

[125] P. Cicuta, E. J. Stancik, and G. G. Fuller, "Shearing or compressing a soft glass in 2D: time-concentration superposition," Physical Review Letters, vol. 90, no. 23, Article ID 236101, 2003.

[126] V. Trappe and D. A. Weitz, "Scaling of the viscoelasticity of weakly attractive particles," Physical Review Letters, vol. 85, no. 2, pp. 449-452, 2000.

[127] L. Imperiali, K.-H. Liao, C. Clasen, J. Fransaer, C. W. MacOsko, and J. Vermant, "Interfacial rheology and structure of tiled graphene oxide sheets," Langmuir, vol. 28, no. 21, pp. 79908000, 2012.

[128] S. Barman and G. F. Christopher, "Simultaneous interfacial rheology and microstructure measurement of densely aggregated particle laden interfaces using a modified double wall ring interfacial rheometer," Langmuir, vol. 30, no. 32, pp. 9752-9760, 2014.

[129] S. Reynaert, P. Moldenaers, and J. Vermant, "Interfacial rheology of stable and weakly aggregated two-dimensional suspensions," Physical Chemistry Chemical Physics, vol. 9, no. 48, pp. 6463-6475, 2007.

[130] C. M. Wijmans and E. Dickinson, "Simulation of interfacial shear and dilatational rheology of an adsorbed protein monolayer modeled as a network of spherical particles," Langmuir, vol. 14 , no. 25 , pp. $7278-7286,1998$.

[131] B. Madivala, J. Fransaer, and J. Vermant, "Self-assembly and rheology of ellipsoidal particles at interfaces," Langmuir, vol. 25, no. 5, pp. 2718-2728, 2009.

[132] M. G. Basavaraj, G. G. Fuller, J. Fransaer, and J. Vermant, "Packing, flipping, and buckling transitions in compressed monolayers of ellipsoidal latex particles," Langmuir, vol. 22, no. 15, pp. 6605-6612, 2006.
[133] E. Brown, H. Zhang, N. A. Forman et al., "Shear thickening and jamming in densely packed suspensions of different particle shapes," Physical Review E, vol. 84, no. 3, Article ID 031408, 2011.

[134] Z. Cui, "Weakly sheared active suspensions: hydrodynamics, stability, and rheology," Physical Review E, vol. 83, no. 3, Article ID 031911, 2011.

[135] E. J. Stancik, M. J. O. Widenbrant, A. T. Laschitsch, J. Vermant, and G. G. Fuller, "Structure and dynamics of particle monolayers at a liquid-liquid interface subjected to extensional flow," Langmuir, vol. 18, no. 11, pp. 4372-4375, 2002.

[136] H. J. Wilson and R. H. Davis, "Shear stress of a monolayer of rough spheres," Journal of Fluid Mechanics, vol. 452, pp. 425441, 2002.

[137] D. S. Keller and D. V. Keller Jr., "The effect of particle size distribution on the antithixotropic and shear thickening properties of coal-water dispersions," Journal of Rheology, vol. 35, no. 8, p. 1583, 1991.

[138] D. Zang, D. Langevin, B. P. Binks, and B. Wei, "Shearing particle monolayers: strain-rate frequency superposition," Physical Review E, vol. 81, no. 1, Article ID 011604, 2010.

[139] H. M. Wyss, K. Miyazaki, J. Mattsson, Z. Hu, D. R. Reichman, and D. A. Weitz, "Strain-rate frequency superposition: a rheological probe of structural relaxation in soft materials," Physical Review Letters, vol. 98, no. 23, Article ID 238303, 2007.

[140] S. Vandebril, J. Vermant, and P. Moldenaers, "Efficiently suppressing coalescence in polymer blends using nanoparticles: role of interfacial rheology," Soft Matter, vol. 6, no. 14, pp. 3353$3362,2010$.

[141] A. Maestro, O. S. Deshmukh, F. Mugele, and D. Langevin, "Interfacial assembly of surfactant-decorated nanoparticles: on the rheological description of a colloidal 2D glass," Langmuir, vol. 31, no. 23, pp. 6289-6297, 2015.

[142] M. Maas, C. C. Ooi, and G. G. Fuller, "Thin film formation of silica nanoparticle/lipid composite films at the fluid-fluid interface," Langmuir, vol. 26, no. 23, pp. 17867-17873, 2010.

[143] P. Degen, M. Paulus, M. Maas et al., "In situ observation of $\gamma$ $\mathrm{Fe}_{2} \mathrm{O}_{3}$ nanoparticle adsorption under different monolayers at the air/water interface," Langmuir, vol. 24, no. 22, pp. 12958 12962, 2008.

[144] P. Degen, D. C. F. Wieland, S. Leick, M. Paulus, H. Rehage, and M. Tolan, "Effect of magnetic nanoparticles on the surface rheology of surfactant films at the water surface," Soft Matter, vol. 7, no. 17, pp. 7655-7662, 2011.

[145] B. P. Binks, Ed., Modern Aspects of Emulsion Science, The Royal Society of Chemistry, Cambridge, UK, 1998.

[146] A. Maestro, E. Guzmán, and E. Santini, "Interfacial rheology of particle-laden interfaces and its role in the stabilization of dispersed systems," in Rheology: Principles, Applications and Environmental Impacts, E. Karpushkin, Ed., pp. 1-26, Nova, New York, NY, USA, 2015.

[147] K. Stratford, R. Adhikari, I. Pagonabarraga, J.-C. Desplat, and M. E. Cates, "Colloidal jamming at interfaces: a route to fluidbicontinuous gels," Science, vol. 309, no. 5744, pp. 2198-2201, 2005.

[148] T. S. Horozov, R. Aveyard, J. H. Clint, and B. Neumann, "Particle zips: vertical emulsion films with particle monolayers at their surfaces," Langmuir, vol. 21, no. 6, pp. 2330-2341, 2005.

[149] D. Zabiegaj, E. Santini, E. Guzmán et al., "Nanoparticle laden interfacial layers and application to foams and solid foams," Colloids and Surfaces A: Physicochemical and Engineering Aspects, vol. 438, pp. 132-140, 2013. 

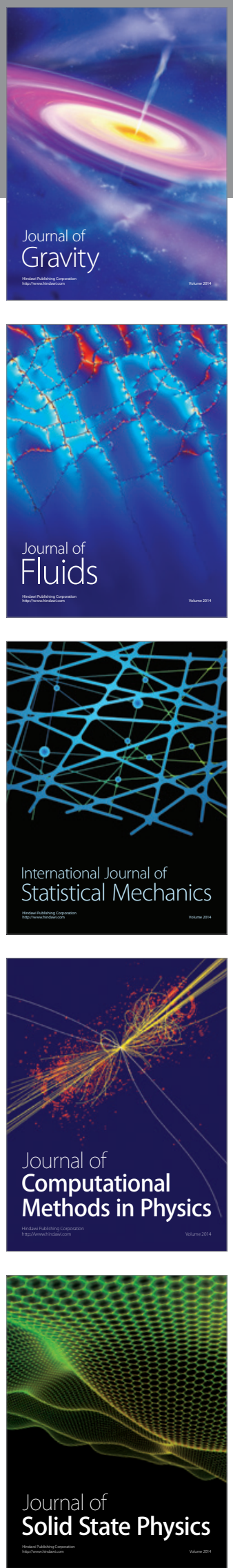

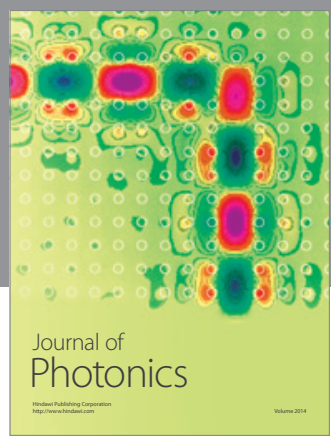

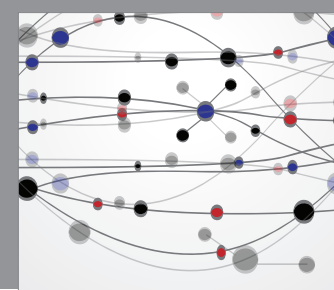

The Scientific World Journal

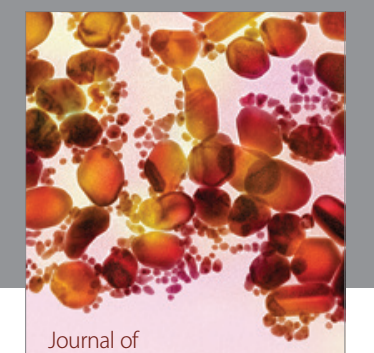

Soft Matter
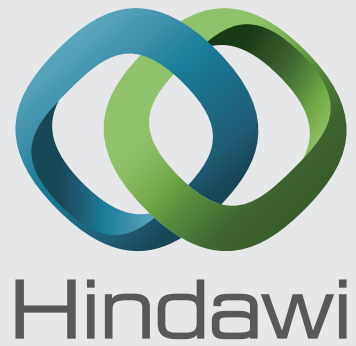

Submit your manuscripts at

http://www.hindawi.com
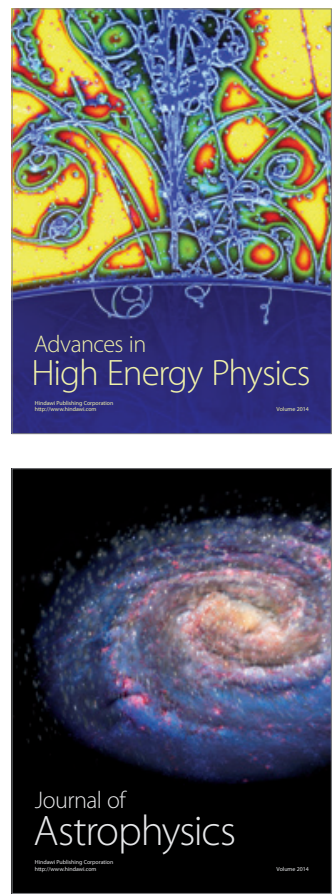
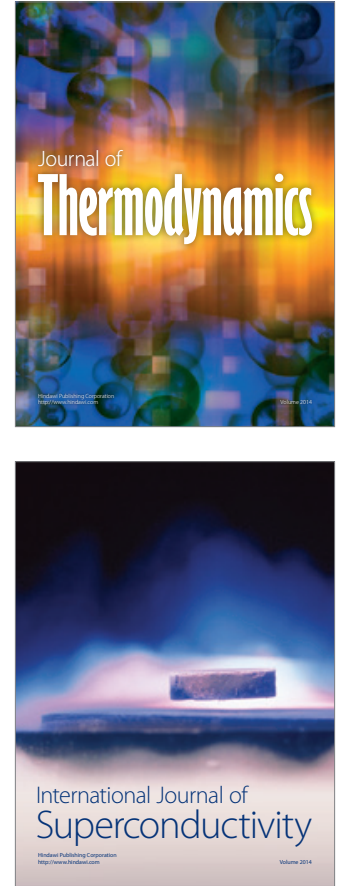
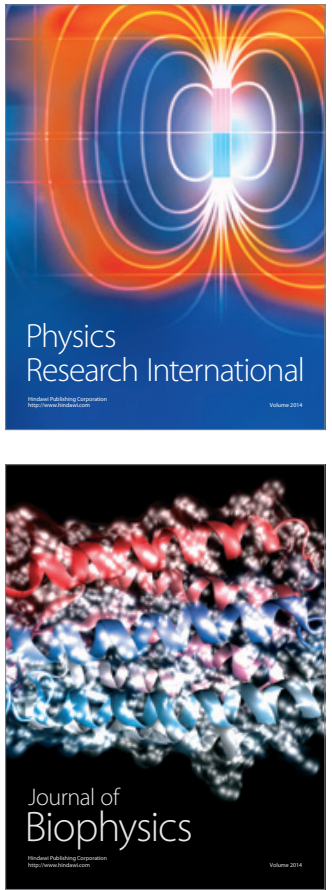
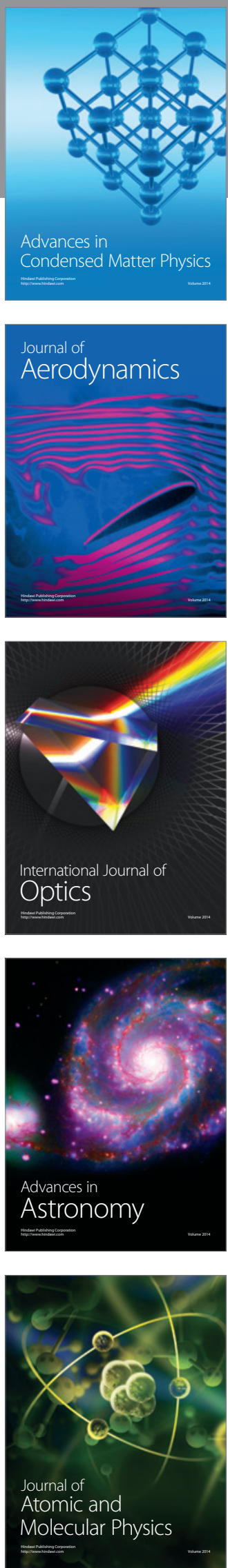